\title{
Fluorescent aminoglycosides reveal intracellular trafficking routes in mechanosensory hair cells
}

\author{
Dale W. Hailey, ${ }^{1,2,3}$ Robert Esterberg, ${ }^{1,2}$ Tor H. Linbo, ${ }^{1}$ Edwin W. Rubel, ${ }^{2,4,5}$ and David W. Raible ${ }^{1,2}$ \\ 'Department of Biological Structure, ${ }^{2}$ Virginia Merrill Bloedel Hearing Research Center, ${ }^{3}$ Carvey Cell Imaging Lab, Institute for Stem Cell and Regenerative Medicine, \\ ${ }^{4}$ Department of Otolaryngology-Head and Neck Surgery, and 5 Department of Physiology and Biophysics, University of Washington, Seattle, Washington, USA.
}

\begin{abstract}
Aminoglycosides (AGs) are broad-spectrum antibiotics that are associated with kidney damage, balance disorders, and permanent hearing loss. This damage occurs primarily by killing of proximal tubule kidney cells and mechanosensory hair cells, though the mechanisms underlying cell death are not clear. Imaging molecules of interest in living cells can elucidate how molecules enter cells, traverse intracellular compartments, and interact with sites of activity. Here, we have imaged fluorescently labeled AGs in live zebrafish mechanosensory hair cells. We determined that AGs enter hair cells via both nonendocytic and endocytic pathways. Both routes deliver AGs from the extracellular space to lysosomes, and structural differences between AGs alter the efficiency of this delivery. AGs with slower delivery to lysosomes were immediately toxic to hair cells, and impeding lysosome delivery increased AG-induced death. Therefore, pro-death cascades induced at early time points of AC exposure do not appear to derive from the lysosome. Our findings help clarify how AGs induce hair cell death and reveal properties that predict toxicity. Establishing signatures for AG toxicity may enable more efficient evaluation of AG treatment paradigms and structural modifications to reduce hair cell damage. Further, this work demonstrates how following fluorescently labeled drugs at high resolution in living cells can reveal important details about how drugs of interest behave.
\end{abstract}

\section{Introduction}

Aminoglycosides (AGs) are broad-spectrum antibiotics that kill aerobic Gram-negative bacteria and mycobacteria (e.g., Mycobacterium tuberculosis). AG treatments are known to damage both the kidney and mechanosensory hair cells (HCs) of the inner ear, with the latter resulting in hearing and balance disorders. Structural modifications and improved treatment paradigms have reduced the incidence of side effects, but kidney damage and inner ear damage still occur, and AGs are contraindicated in a number of scenarios where they would otherwise be useful. Nonetheless, AGs remain a primary treatment in many life-threatening situations, including Pseudomonas infection, multi-drug-resistant tuberculosis, neonatal sepsis, complicated urinary tract infections, and endocarditis (1-3). While the incidence of kidney damage can be reduced with modified dosing paradigms, inner ear damage remains a common and permanent side effect of AG treatment.

Though it has been known for well over 50 years that AGs cause hearing loss, the mechanisms that underlie AG-induced HC toxicity are still debated. Previous studies implicate a number of possibilities. In prokaryotes, antimicrobial activities of AGs have been attributed to binding of the $30 \mathrm{~S}$ ribosomal subunit (4-6). In eukaryotic cells, AG toxicity may occur through interactions with the related $12 \mathrm{~S}$ mitochondrial ribosomal RNA subunit (7). This is a parsimonious explanation for why mutations in mitochondrial ribosomal RNAs can severely sensitize patients to AG exposure. Other

Conflict of interest: The authors have declared that no conflict of interest exists. Submitted: October 9, 2015; Accepted: November 3, 2016. Reference information: J Clin Invest. 2017;127(2):472-486. https://doi.org/10.1172/JCI85052. studies suggest that AG interactions with the plasma membranespecifically affecting metabolic regulation of phosphoinositides cause cell damage $(8,9)$. A wealth of reports indicate that AGs activate cell death cascades through diverse signaling pathways (10, $11)$, and both caspase-dependent $(12,13)$ and -independent (14) routes are implicated. Our recent studies suggest that AG-induced calcium flow between endoplasmic reticulum and mitochondria results in reactive oxygen species and subsequent cell death (15, 16). The many reported activities of AGs make it challenging to identify clear targets to block AG-induced HC death (17).

One fundamental property of cells that are vulnerable to AG-induced damage is their tendency to take up and retain AGs. While AGs are rapidly cleared from serum, both kidney proximal tubule and inner ear HCs accrue AG antibiotics (17). It has been proposed that the main entry of AGs into kidney cells is through endocytosis $(18,19)$. However, enrichment of AGs in HCs requires mechanotransduction (MET) (20). These studies suggest that AGs may enter through several routes, each of which may have different toxicity outcomes.

We examined intracellular trafficking of different AGs and asked whether altering those pathways influences their relative toxicity. We followed labeled AGs in live functioning HCs in the zebrafish lateral line (LL) sensory system. LL sensory HCs of larval zebrafish are convenient for these types of studies because they reside externally on the head and body surface of larval zebrafish (21). The LL informs nonmammalian aquatic vertebrates about proximal movements in the water. These movements are detected by superficial mechanosensory HC clusters that are innervated by the distal ends of afferent fibers that project to the hindbrain $(22,23)$. LL HCs are morphologically and 

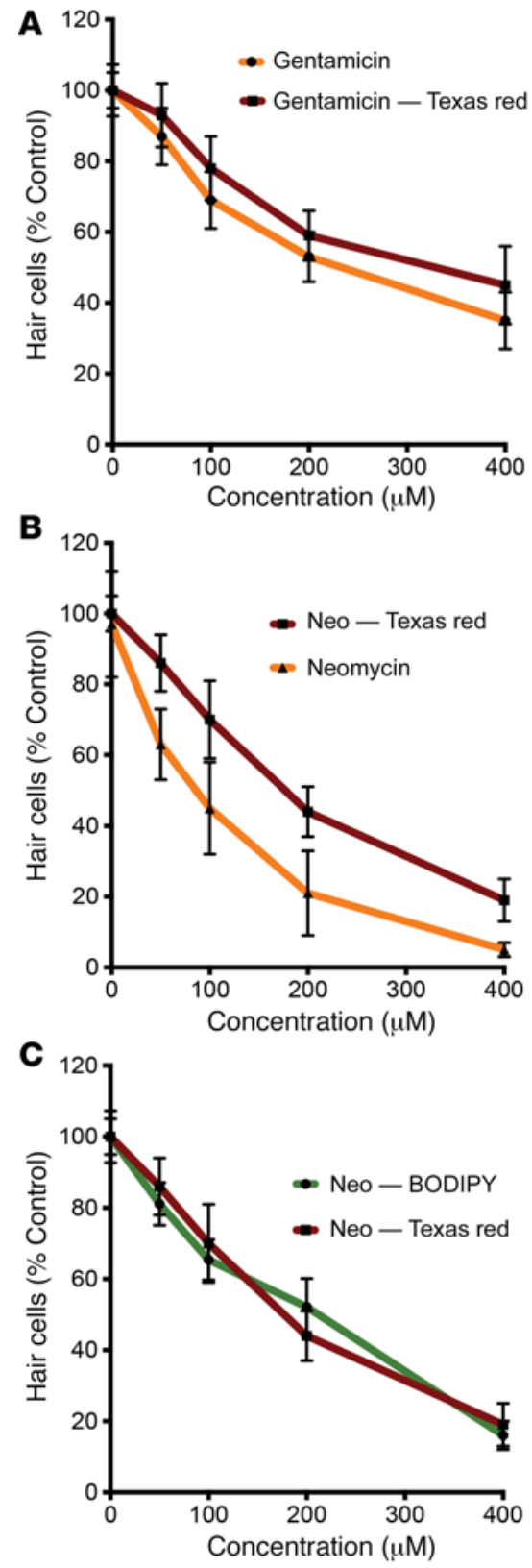
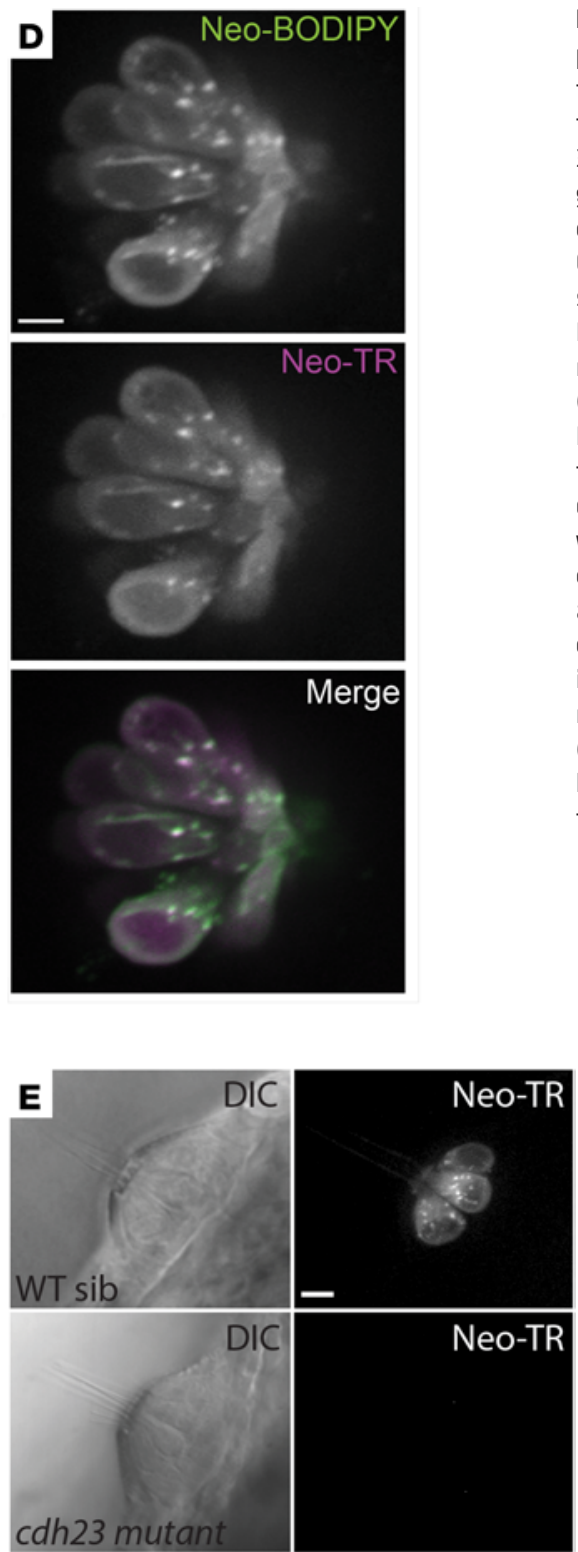

Figure 1. Fluorescently labeled AGs are effective proxies for unlabeled AGs. (A) Dose-response functions for gentamicin and Gent-TR. Zebrafish 5 days after fertilization were treated for 30 minutes with indicated concentrations of gentamicin or Gent-TR. Following a 1-hour recovery, surviving HC nuclei were labeled with SYTOX Green (ThermoFisher) and counted. Each graphed symbol represents 5 fish, 5 neuromasts per fish. Percentages indicate $\mathrm{HCs}$ that survived the treatment relative to $\mathrm{HCs}$ present in untreated fish. (B) Dose-response functions for neomycin and Neo-TR. (As described for A.) (C) Dose-response functions for Neo-BODIPY and Neo-TR. (As described for A.) (D) Overlap of signals from AGs with structurally distinct fluorescent tags. Image of $\mathrm{HCs}$ pulse-labeled with a mix of $25 \mu \mathrm{M}$ Neo-TR and $25 \mu \mathrm{M}$ Neo-BODIPY. (E) Labeled AGs do not enter HCs that lack MET activity. Differential interference contrast and fluorescent images of a neuromast in a WT sibling (top) or a sputnik ${ }^{\text {ti264a }}$ (cdh23 loss-of-function mutant) zebrafish that lacks MET (bottom). Scale bars: $5 \mu \mathrm{m}$. Error bars for all plots: $\pm 1 \mathrm{SD}$. structurally similar to HCs in the mammalian inner ear, and are sensitive to drugs and small molecules known to cause hearing loss in humans (e.g., the AGs, cisplatin, and copper) (24-28). Our results demonstrate distinct pathways of AG uptake that appear to confer different levels of toxicity, and further suggest that altering AG trafficking can protect HCs from damage. Signatures for less toxic AGs may also facilitate screening for new AGs that are now being developed (29).

\section{Results}

Vetting tagged AGs. To follow entry and intracellular trajectories of AGs, we made AGs that incorporated a fluorescent tag. The use of fluorescent analogs of AGs is well established. Previous studies using gentamicin tagged with the fluorescent moiety Texas red (Gent-TR) revealed delivery routes taken by AGs to the mouse inner ear. Gent-TR injected into mice recapitulates the localization of gentamicin labeled by immunohistochemistry, and is toxic to HCs (30). Furthermore, unconjugated gentamicin competes with Gent-TR, supporting its use as a proxy for the unlabeled form (31). Here we used Texas red labeling to follow the behavior of AGs as they enter and distribute to intracellular compartments in zebrafish LL HCs.

To validate the labeled AGs, we assessed their toxicity and specificity. Supplemental Video 1 (supplemental material available online with this article; doi:10.1172/JCI85052DS1) shows the very specific enrichment of Texas red-labeled neomycin (Neo-TR) in functional LL HCs. To assess toxicity, we generated dose-response functions for both labeled and unlabeled forms a standard approach in zebrafish HC studies (32). Dose-response functions revealed that tagged AGs show a small decrease in toxicity but retain characteristics of their respective unlabeled compounds. The toxicity of both neomycin and Neo-TR is rapid and 
A
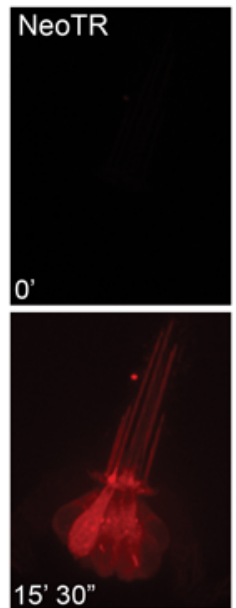

$15^{\prime} 30^{\prime \prime}$

B
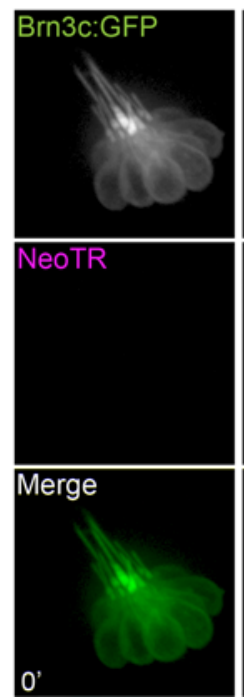
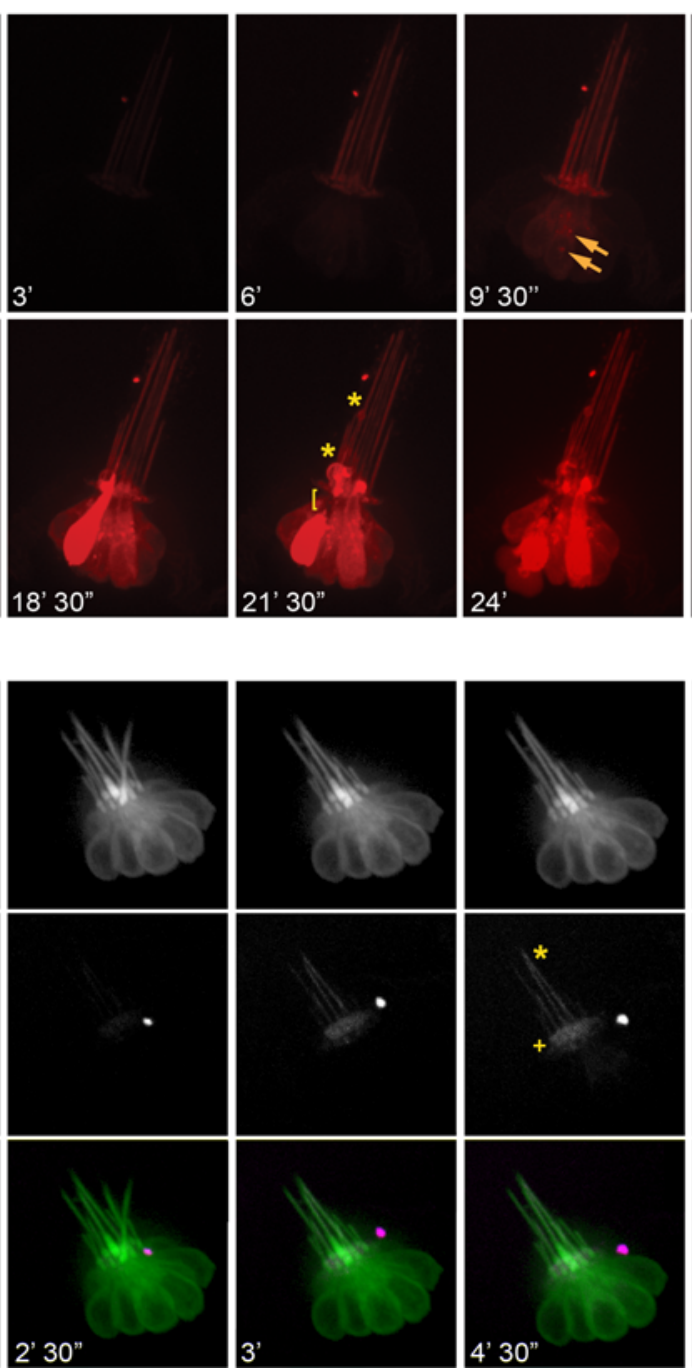
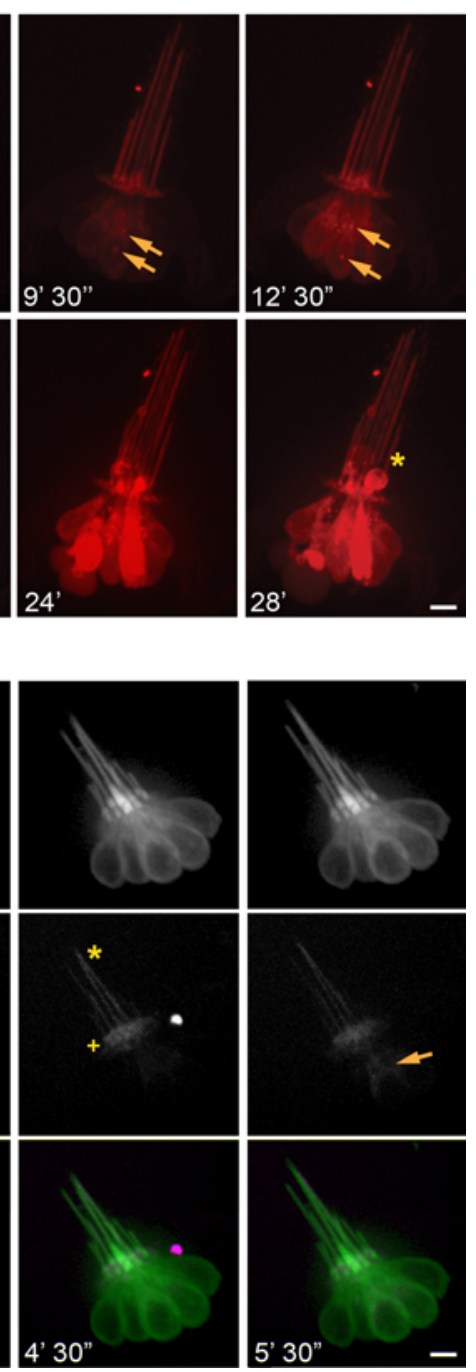
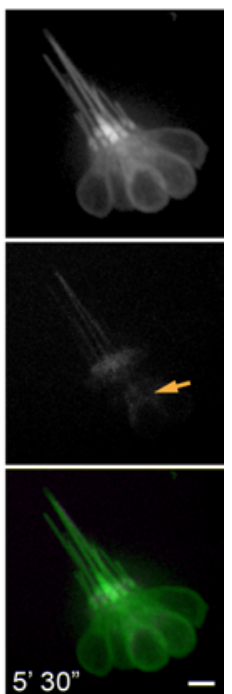

Figure 2. Fluorescent neomycin preferentially enters and kills LL HCs. (A) A neuromast in a sedated 5 days post fertilization (dpf) zebrafish imaged during chronic exposure to 50 $\mu \mathrm{M}$ Neo-TR. Neo-TR was added to the media at time 0 . Neo-TR first enters the kinocilia and stereocilia (6 minutes) and subsequently the $\mathrm{HC}$ body in both puncta (yellow arrows) and diffuse pools ( 9 minutes 30 seconds). Note that non-HCs in the fish do not take up Neo-TR. HCs with high concentrations of Neo-TR show blebbing at the apical region and kinocilia (asterisks: 21 minutes 30 seconds; 28 minutes), and separation of the apical region from the HC body (bracket: 21 minutes $30 \mathrm{sec}-$ onds). (See also Supplemental Video 3.) Scale bar: $10 \mu \mathrm{m}$. (B) Neo-TR time series in a brn3cGFP transgenic zebrafish, treated as in $\mathbf{A}$. The appearance of Neo-TR on the stereocilia (plus symbol) and kinocilia (asterisk) occurs nearly simultaneously and prior to detectable Neo-TR throughout the HC body (yellow arrow). Scale bar: $10 \mu \mathrm{m}$. (See also Supplemental Video 3.) extensive. Comparable short-term exposures to gentamicin and Gent-TR damage a smaller fraction of the HCs, consistent with previous work characterizing the different toxicity profiles of the unlabeled forms of these AGs (ref. 33 and Figure 1, A and B). To further address whether addition of Texas red affects the behavior of conjugated AGs, we evaluated AGs tagged with another fluorescent moiety, BODIPY, that is structurally unlike Texas red. Conjugation with BODIPY does not alter dose-response characteristics (Figure 1C), and AG-BODIPY conjugates completely colocalize with $\mathrm{AG}^{-}$Texas red conjugates in exposed $\mathrm{HCs}$ (Figure 1D). Like unconjugated AGs, fluorescently tagged AGs readily enter HCs, and their entry is abolished in HCs that lack MET activity, such as the cadherin 23 (cdh23) mutant sputnik $k^{\text {t264a }}$ (Figure 1E and refs. 34, 35). This observation is consistent with reports showing that MET activity is necessary for AG HC toxicity $(20,36)$. Additionally, neither unconjugated Texas red nor unconjugated BODIPY alone enters HCs (Supplemental Figure 1). Lastly, unlabeled AGs compete with labeled AGs in uptake assays (Supplemental Figure 2). Taken together, these data indicate that Texas red- and BODIPY-conjugated AGs are reasonable proxies for unlabeled AGs, supporting their use as tools for studying the behaviors of AGs within LL HCs.
Characterizing entry and intracellular distribution of AGs. To visualize AG entry and distribution in LL HCs, we first monitored HCs in 5 days post fertilization (dpf) zebrafish that were chronically exposed to Neo-TR. Zebrafish were anesthetized with tricaine, immobilized under mesh, and imaged on an inverted microscope. During the course of iterative imaging, $50 \mu \mathrm{M}$ Neo-TR was added to the media (Figure 2A and Supplemental Video 2). Within 5 minutes of exposure, Neo-TR appears in the apical region of HCs, in both the kinocilia and the stereocilia. The labeling of these 2 apical compartments occurs simultaneously (Figure 2B and Supplemental Video 3). While this rapid labeling may indicate AG interaction with the outer leaflet of apical membranes, kinocilia labeling does not disappear when Neo-TR is washed out, and labeling of the kinocilia and stereocilia is orders of magnitude brighter than any other cell labeling on the surface of the fish. Labeling of kinocilia also requires active MET, suggesting that labeling is not simply a "sticky" interaction. Stereocilia labeling is consistent with transit through MET channels reported to be in the stereocilia, but why kinocilia labeling occurs is unclear at this time. Notably, recent work shows that mutations in several kinocilia proteins affect AG toxicity (37). Following appearance in the apical HC 
A
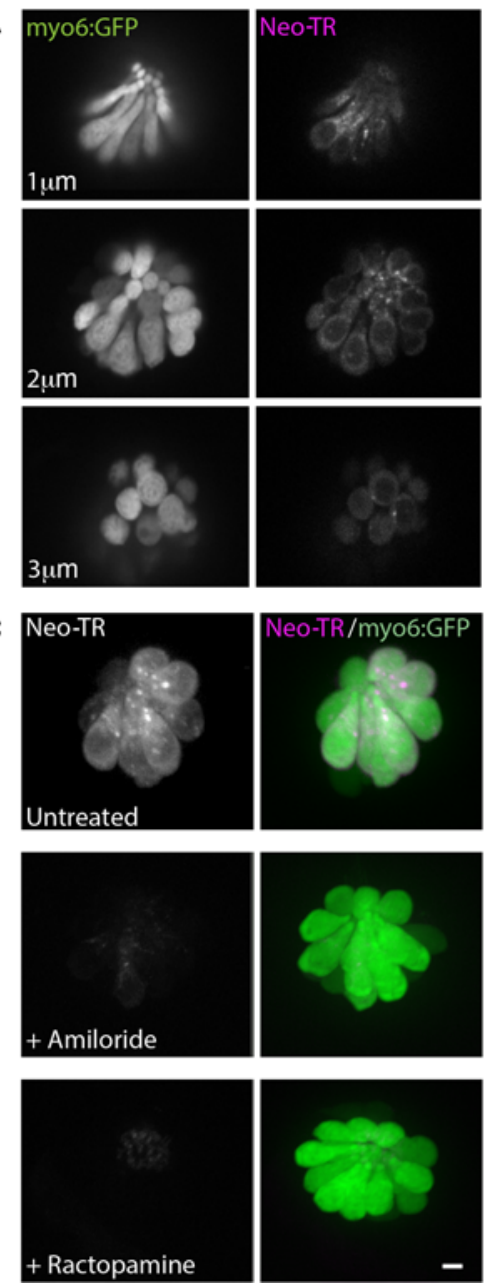
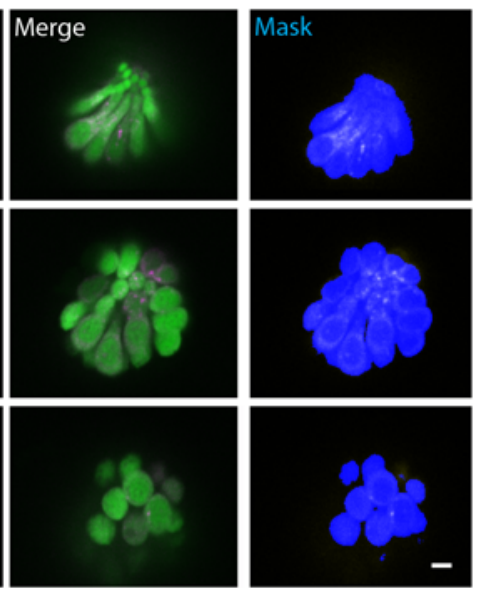

D

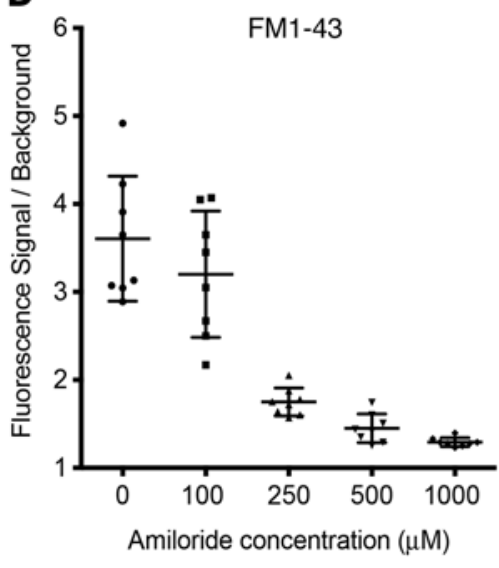

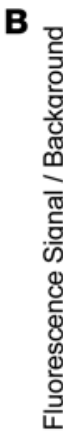

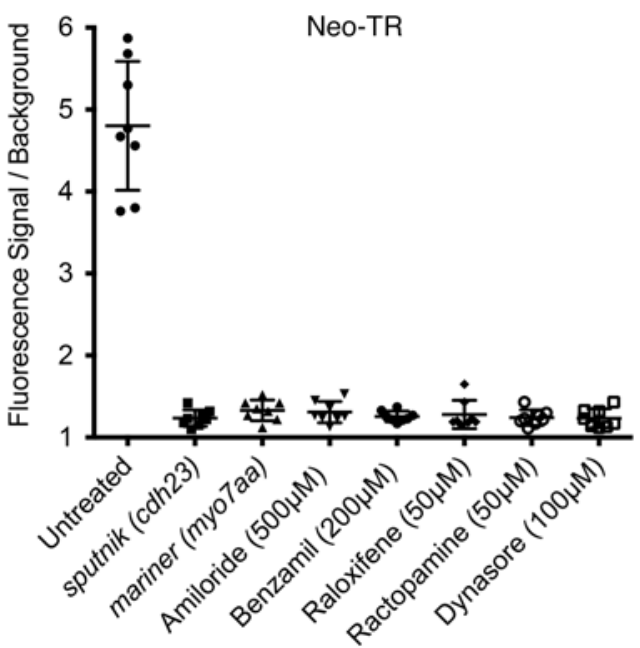

E

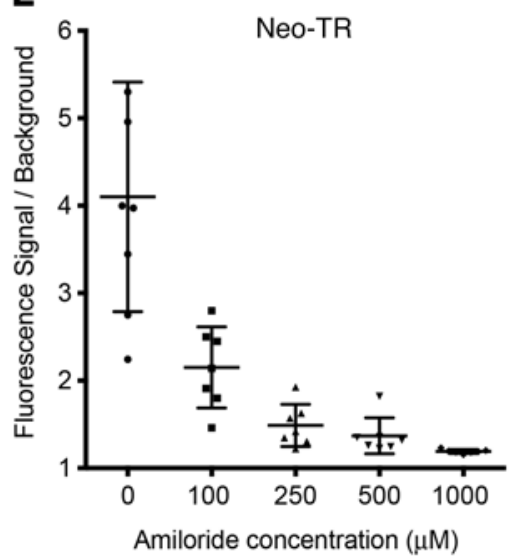

Figure 3. Small molecules and mutations protect HCs from AG exposure by affecting MET and AG loading. (A) Example images from a Z-series showing technique to quantify AG loading. myo6-GFP zebrafish have labeled LL HCs (left panels). Otsu segmentation of the GFP signal generates an HC-specific mask (right panels). This is used to quantify the Neo-TR signal in HCs (middle left panels). Scale bar: $5 \mu \mathrm{m}$. (B) Diverse types of small molecules protect HCs from AG exposure by inhibiting AG entry, as is seen in the sputnik ${ }^{\mathrm{t} 264 a}$ and mariner ${ }^{\mathrm{t} y 220} \mathrm{MET}$ mutants. Neo-TR uptake is quantified as described in $\mathbf{A}$. Each graphed symbol represents 1 fish, 5 neuromasts per fish. (C) Example images from Neo-TR pulse-labeled HCs either untreated, treated with the MET inhibitor amiloride, or treated with ractopamine. Scale bar: $5 \mu \mathrm{m}$. (D) The MET inhibitor amiloride impedes HC entry of the MET activity indicator FM1-43 in a dose-dependent manner. Zebrafish ( $5 \mathrm{dpf}$ ) were pretreated with amiloride at the indicated concentrations for 5 minutes, exposed to amiloride plus 500 nM FM1-43 for 5 minutes, washed 3 times, and imaged. FM1-43 fluorescence in neuromasts was quantified and expressed as signal relative to background outside HCs. (E) The dose-dependent reduction of Neo-TR loading mimics the reduction in MET activity in D. (Quantified as in D, but with $50 \mathrm{nM}$ Neo-TR replacing FM1-43.) Error bars for all plots: \pm 1 SD.

region, Neo-TR enters the cell body, where it is present in diffuse and punctate pools. Intensity in the puncta is strikingly higher than in the diffuse pool, indicating that AGs are concentrated in these structures. Signals in both the diffuse and punctate pools steadily increase during chronic exposure. In the subset of HCs that are killed by $50 \mu \mathrm{M}$ Neo-TR, diffuse Neo-TR signal abruptly rises just before HC death. We suggest that this abrupt rise corresponds with the loss of membrane integrity that occurs before death (15). All labeled AGs that we monitored show these same properties - namely, the initial appearance at the apical region, the movement into diffuse and punctate pools, and the increased diffuse signal just before cell death.

Quantifying AG loading. To quantify AG loading into HCs, we exposed active unsedated zebrafish to a 5-minute pulse of 50 $\mu \mathrm{M}$ Neo-TR, washed out the zebrafish, and immediately imaged neuromasts. These experiments were done in a zebrafish line that expresses cytosolic GFP in HCs. We wrote a MATLAB script to identify the GFP-positive HC volume using automated Otsu thresholding on 16-bit image Z-stacks $(38,39)$. AG-Texas red signal was then calculated within this HC volume (Figure 3A). Data are shown as the average Neo-TR signal within the neuromast volume divided by the average non-neuromast Neo-TR background signal. Consistent with the reported requirement for MET activity for AG loading, mutants that lack MET activity (the cadherin 23 mutant sputnik ${ }^{\text {tj264a }}$ and the myosin VIIAa [myo7aa] mutant marinerty220; refs. 35,40 ) show a striking reduction in Neo-TR loading to near background levels (Figure 3B). In zebrafish treated with the known MET activity inhibitors amiloride and benzamil (41), Neo-TR was reduced to levels comparable to those in the MET activity mutants (Figure 3B). 
A
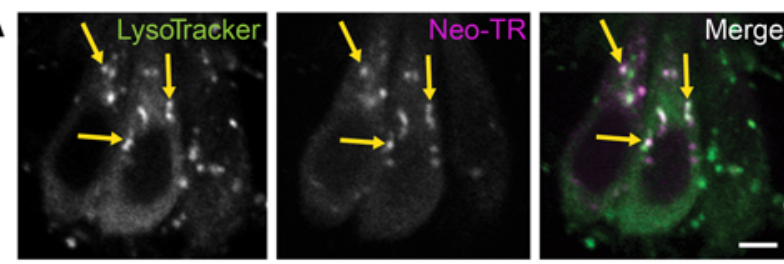

B
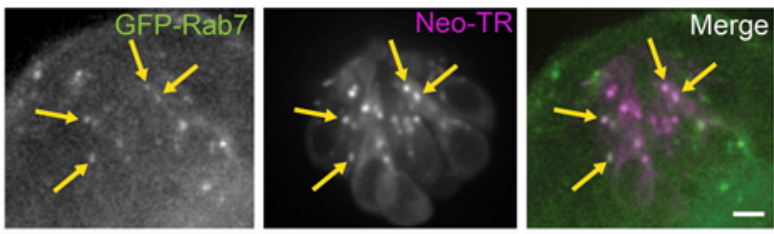

C

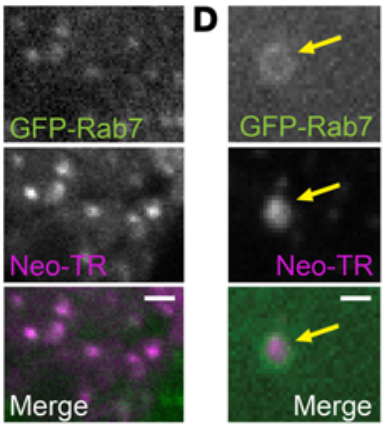

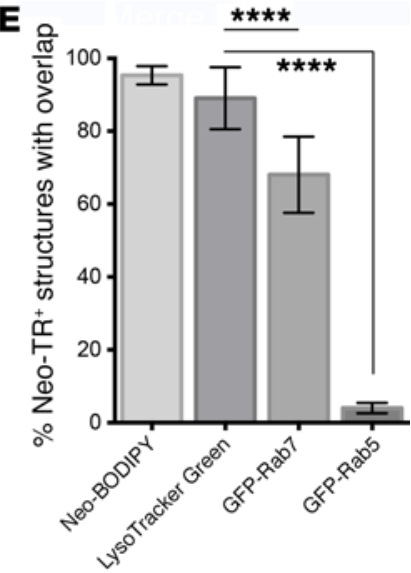

Figure 4. Fluorescent AGs label lysosomes. (A) Neo-TR-labeled puncta in exposed HCs also label with lysosomal markers. $5 \mathrm{dpf}$ zebrafish were simultaneously exposed to $50 \mu \mathrm{M}$ Neo-TR and LysoTracker Green before washout and imaging. Most LysoTracker-positive puncta contain Neo-TR (see yellow arrows as examples). Note that some immature HCs label with LysoTracker but not appreciable Neo-TR (right cell). Scale bar: $5 \mu \mathrm{m}$. (B) Neo-TR puncta colocalize with a genetically encoded late endosome/ lysosome marker, GFP-Rab7. Transgenic zebrafish ubiquitously expressing GFP-Rab7 were exposed to Neo-TR. Most Neo-TR-positive puncta also have accumulations of GFP-Rab7 (see yellow arrows as examples). Scale bar: $5 \mu \mathrm{m}$. (C) Zoom of an intracellular region of an HC with GFP-Rab7/ Neo-TR double-labeled puncta. Scale bar: $3 \mu \mathrm{m}$. (D) Neo-TR is present in the lumen of lysosomes. In large lysosomes where the outer membrane and lumen can be resolved, GFP-Rab7 marks membranes that surround Neo-TR signal within the lumen. Scale bar: $2 \mu \mathrm{m}$. (E) Quantification of overlap of markers with Neo-TR signal in puncta. Neo-BODIPY and GFPRab5 show results of markers where there is either near-complete or near-absent overlap (error bars: $\pm 1 \mathrm{SD}$ ). Data taken from 10 neuromasts. ${ }^{* * *} P<0.0001$ based on Holm-Šidák multiple comparison analysis after Kruskal-Wallis test. (See Figure 1 and Supplemental Figure 3C for representative Neo-BODIPY and GFP-Rab5 images.)

Using this technique, it is straightforward to quantitatively evaluate whether other compounds previously shown to protect HCs from AG exposure affect AG loading. Many such compounds have been identified in LL zebrafish screens (42-44). Here we show results for benzamil, raloxifene, ractopamine, and dynasore. All of these compounds reduce loading to levels seen when MET activity is absent or blocked (Figure 3B). Images comparing untreated, amiloride-treated, and ractopamine-treated neuromasts are shown in Figure 3C. Compounds that inhibit AG loading comprise a diverse set of functions; here, compounds reported as sodium channel blockers (amiloride and benzamil), a selective estrogen receptor modulator (raloxifene), an epinephrine mimetic (ractopamine), and a dynamin inhibitor (dynasore) all strongly inhibit AG loading. The loading of AGs into HCs is sensitive to a diverse array of small molecules that do not share an obvious pharmacological target. In our studies to date, protective compounds like benzamil, raloxifene, ractopamine, and dynasore that inhibit AG entry also inhibit FM1-43 uptake (data shown for amiloride in Figure 3E and benzamil discussed below), consistent with MET activity being critical for AG loading, as has been reported in other systems (20).

To characterize the relationship between MET activity and loading of AGs, we evaluated Neo-TR and FM1-43 loading in zebrafish mutants treated with a range of amiloride concentrations. FM1-43 entry through MET channels depends on the open probability of the channels (45), and we were able to identify a range of amiloride concentrations $(0$ to $1,000 \mu \mathrm{M})$ that demon- strate dose-dependent effects of amiloride on FM1-43 entry (Figure 3D). This same range of amiloride concentrations shows a graded effect on Neo-TR entry into LL HCs (Figure 3E).

Characterizing puncta. Treatment with compounds at concentrations that appear to completely block AG entry prevents the appearance of both the diffuse pool in the cytosol and the pool present in puncta. However, when the rate of AG entry is delayed but not blocked, these intracellular pools could play differential roles in toxicity. To characterize these different intracellular AG pools, we first sought to identify the Neo-TR puncta that appear shortly after exposure. The reported binding of the $12 \mathrm{~S}$ mitochondrial ribosomal subunit (7) suggests that AGs might accrue in mitochondria. However, mitochondria labeled by either transgenic expression of mitochondrial-targeted GCaMP (15) or the vital dye MitoTracker Green contained no detectable Neo-TR signal (Supplemental Figure 3A). Previous studies using kidney cell culture lines indicate that AGs can transit the secretory system and the Golgi $(46,47)$. However, in LL HCs, we did not find any detectable Neo-TR signal in the Golgi of LL HCs labeled by transgenic expression of mannosidase 2-GFP (Supplemental Figure 3B). Additionally, we saw no effect on the intracellular distributions of Neo-TR when the Golgi organelle was disrupted by brefeldin A treatment (48). Based on reports of endocytic uptake of AGs, we also evaluated Neo-TR localization in the HCs of transgenic zebrafish expressing the early endosome marker GFP-Rab5. Rab5 marks early stages of many endocytic routes, most of which utilize clathrin. Detectable Neo-TR did not appear in Rab5-positive endosomes (Supplemental Figure 3C). Notably, not all early endocytic routes employ Rab5 (49), including routes that 

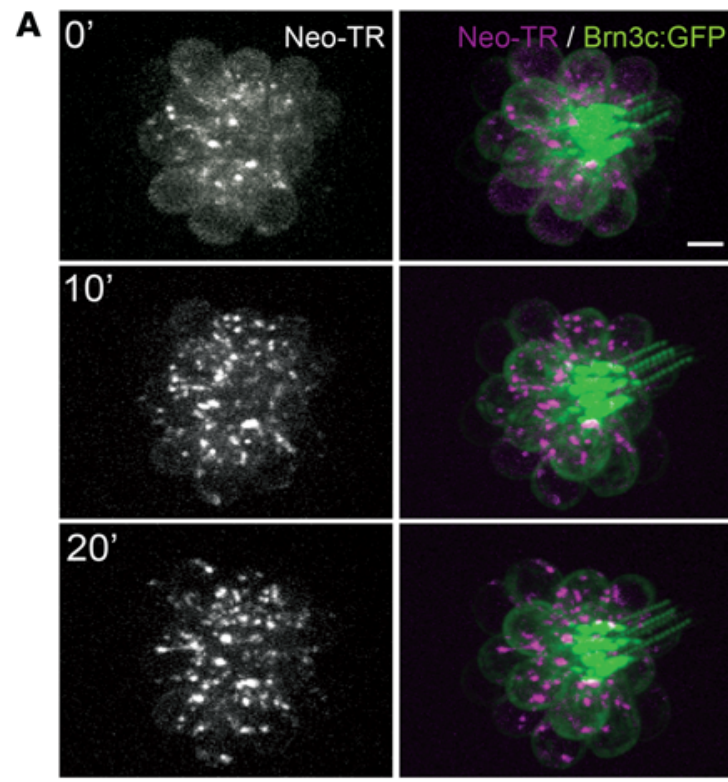

$\mathbf{B}$

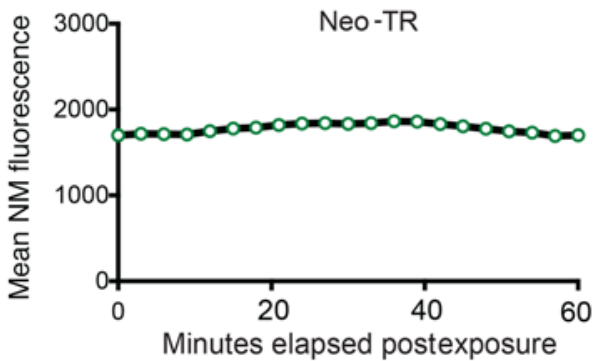

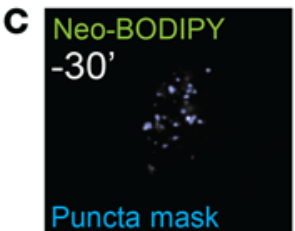
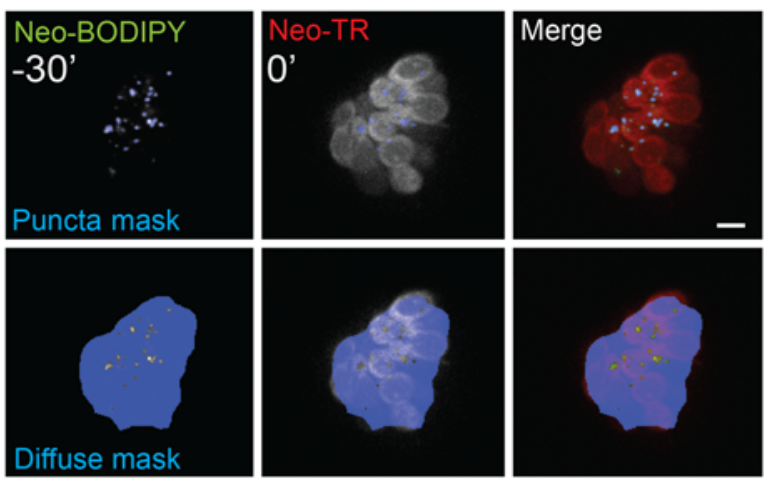

D
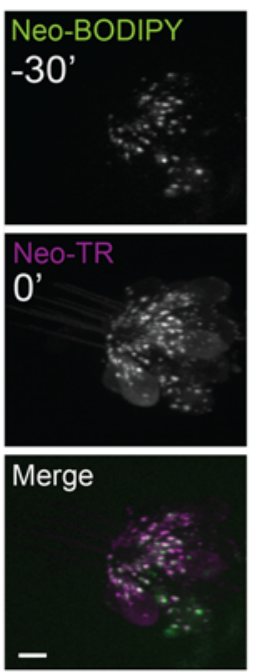

$\mathbf{E}$

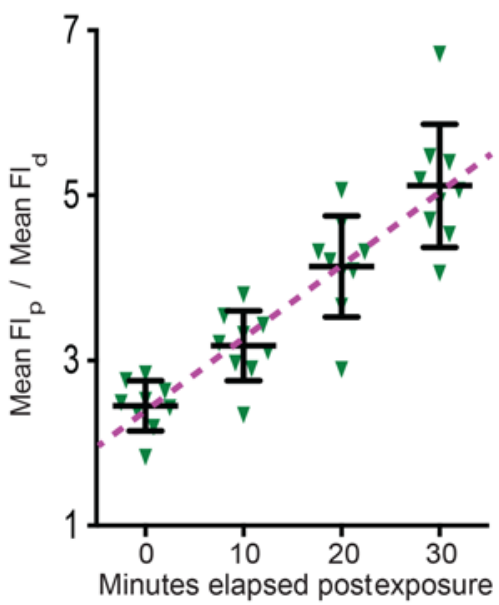

Figure 5. Labeled AGs transit from the diffuse intracellular pool into lysosomes. (A) Time-lapse imaging of Neo-TR in pulse-labeled HCs shows a progressive accumulation of Neo-TR in puncta and loss of diffuse signal in the cytosol. Redistribution is apparent within 20 minutes of exposure. Scale bar: $5 \mu \mathrm{m}$. (See also Supplemental Video 4.) (B) Total intracellular Neo-TR fluorescence following pulse exposure does not decrease, indicating that loss of Neo-TR signal from the cytosol is likely due to redistribution to lysosomes and not export from cells. NM, neuromast. (C) An example of image masks (in blue) used to monitor the lysosomal and cytosolic fractions of Neo-TR. Image captured immediately after Neo-TR exposure. Scale bar: $5 \mu$ m. (D) Neo-TR added 30 minutes after Neo-BODIPY pulse exposure accumulates in the Neo-BODIPY-prelabeled structures. Image captured 10 minutes after Neo-TR exposure. Scale bar: $5 \mu \mathrm{m}$. (E) Quantification of loading into lysosomes following a Neo-TR pulse. After 30 minutes, intensity within the lysosomal mask is approximately 5 -fold higher than intensity in the cytosol. Each graphed symbol represents 1 fish, 3 neuromasts per fish. Error bars: \pm 1 SD. Fl, Fluorescence in puncta; $\mathrm{Fl}_{\mathrm{d}}$, diffuse fluorescence.

utilize dynamin and routes that deliver substrates from the extracellular environment to lysosomes $(50,51)$. Lastly, we evaluated whether lysosomes are enriched in Neo-TR. In HCs of the neonatal chick, accumulations of injected kanamycin were previously found in lysosomes identified by acid phosphatase cytochemistry (52), and dramatic increases in the density of lysosomes following gentamicin exposure are seen in chick hair cells prior to hair cell death $(53,54)$. We assessed whether Neo-TR was present in lysosomes labeled with the vital dye LysoTracker Green. LysoTracker Green consistently labeled puncta that also contained Neo-TR (Figure 4A). Since LysoTracker dyes also label other low-pH organelles like synaptic vesicles (55), we further discriminated whether the puncta were lysosomes by surveying the late endosome/lysosome marker Rab7. Rab7 transiently binds to late endosomal and lysosomal membranes $(56,57)$. In transgenic zebrafish that express GFPRab7 under a ubiquitous promoter, GFP-Rab7 is expressed in all cells (not just HCs), and a large fraction of the protein is cytosolic. This accounts for the prominent diffuse background seen throughout the neuromast. A majority of puncta enriched for GFP-Rab7 in HCs also contained Neo-TR following a pulse exposure (Figure 4B). An enlarged image of just the HC region is shown in Figure 4C. In large GFP-Rab7-labeled lysosomes, we could resolve NeoTR signal inside the lysosomal lumen, demarcated by GFP-Rab7 membrane labeling (Figure 4D). Colocalization of GFP-Rab7 with Neo-TR puncta is apparent from the time we are first able to detect puncta. The degree of overlap of LysoTracker Green and GFPRab7, compared with the degree of overlap with Neo-BODIPY (which we expect to overlap completely) and Rab5 (which does not significantly overlap), is quantified in the graph in Figure 4E. Our results demonstrate that a significant fraction of AGs are retained in lysosomes. This is consistent with previous work in other model systems $(52,58)$. Neo-TR puncta that do not show colocalization with lysosomal markers are most likely endocytic carriers that are not positive for Rab7 and do not have sufficiently low $\mathrm{pH}$ to retain LysoTracker label. At this time, we have not been able to further characterize these endocytic carriers. However, the early appearance of all puncta is blocked when dynamin-dependent endocytosis is inhibited (discussed below). 
A
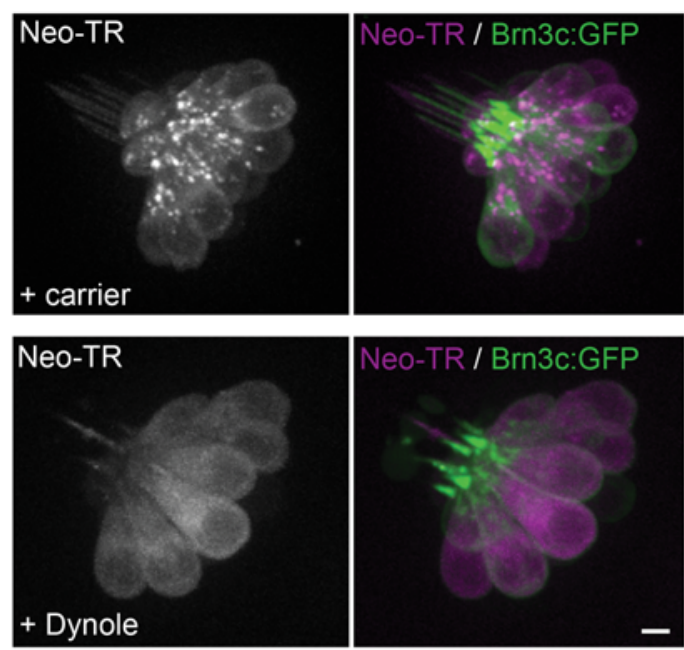

C
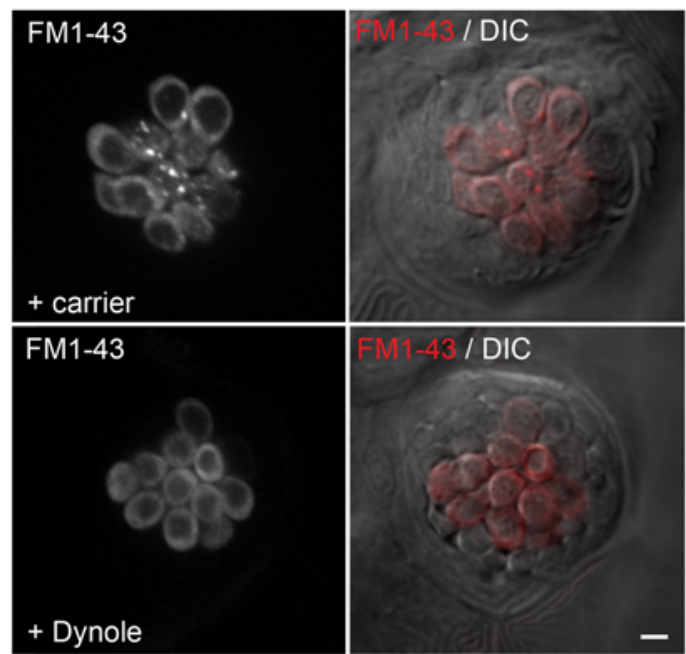

B

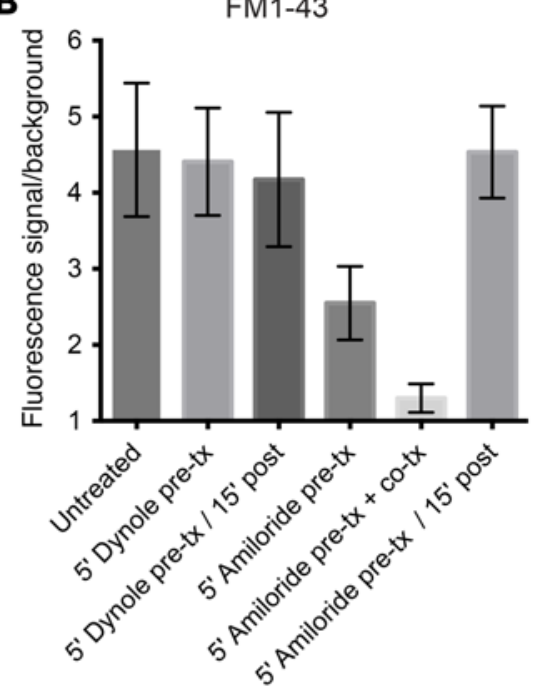

D

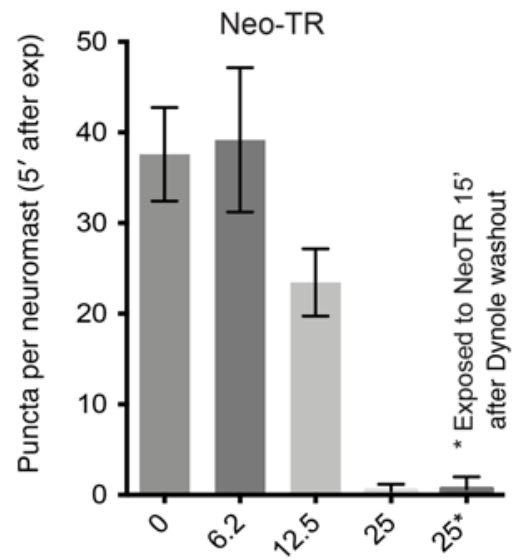

Dynole 34-2 concentration ( $\mu \mathrm{M})$
Figure 6. Inhibiting endocytosis with Dynole prevents the rapid delivery of Neo-TR to lysosomes without affecting MET activity. (A) HCs were exposed to Neo-TR in either the absence (top panels) or presence (bottom panels) of the dynamin inhibitor Dynole 34-2 and imaged immediately after exposure. Inhibition of dynamin activity abolishes rapid appearance of Neo-TR-labeled puncta. Scale bar: $5 \mu \mathrm{m}$. (B) HCs were exposed to a pulse of FM1-43 as described in Figure 3B. Treatment with Dynole 34-2 does not significantly inhibit FM1-43 loading, either immediately or 15 minutes after Dynole pulse exposure. This is in contrast to amiloride treatment, which is also reversible, unlike Dynole 34-2. Each column: 3 fish, 5 neuromasts per fish. (C) Images of HCs exposed to FM1-43 in either the absence or presence of Dynole 34-2. Like Neo-TR, the rapid appearance of FM1-43 puncta is abolished. Scale bar: $5 \mu \mathrm{m}$. (D) Twenty-five micromolar Dynole $34-2$ is sufficient to block the appearance of almost all puncta. Note that Dynole 34-2 activity is not rapidly reversible; 15 minutes after Dynole 34-2 washout, Neo-TR still does not appear in puncta following exposure (far right bar). Error bars: $\pm 1 \mathrm{SD}$. Each graphed symbol represents 3 fish, 5 neuromasts per fish.
Characterizing delivery of AGs into lysosomes. Immediately after pulse exposure and washout, all labeled AGs we evaluated show a prominent diffuse pool present in the cytosol. This diffuse signal decreases with time, and the loss of diffuse signal occurs concurrently with increased signal in lysosomes (Figure 5A; see also Supplemental Video 4). We do not detect a net loss of signal from the entire HC (Figure 5B); the drop in the diffuse signal appears to be the result of delivery of Neo-TR to lysosomes rather than export of Neo-TR from the cell. To quantify movement of Neo-TR from the cytosol to lysosomes, we took advantage of 2 observations: (a) NeoBODIPY and Neo-TR label the same puncta (shown in Figure 1D), indicating that these 2 visually separable compounds are trafficked to the same structures; and (b) lysosomes previously labeled with Neo-BODIPY also label with Neo-TR from a later pulse exposure, indicating that the AGs are delivered to preexisting puncta (Figure $5 \mathrm{D})$. To quantify rates of AG loading into lysosomes, we exposed HCs to a low-concentration $(20 \mu \mathrm{M})$ pulse of Neo-BODIPY for 5 minutes, and washed and transferred zebrafish to fresh medium for an additional 30 minutes. This first Neo-BODIPY pulse marks lysosomes with green signal. We then re-exposed these zebrafish to a pulse of Neo-TR, and asked how quickly green lysosomes acquire red Neo-TR from the second pulse (Figure $5 \mathrm{C}$ ), using the green signal to make an image mask. We quantified the red signal within the green lysosome mask (Figure 5C, top panel), and compared this with the red signal outside the lysosome mask (Figure $5 \mathrm{C}$, bottom panel). We used these values to determine the relative amount of Neo-TR signal in the lysosomes: the mean Neo-TR signal present in the lysosomes divided by the mean Neo-TR signal in HCs excluding the lysosome signal. This ratio steadily increases with time following a pulse exposure to Neo-TR (Figure 5E), which we can represent as a sloped line (dashed line, Figure 5E). We use the slope of the line representing change from 0 to 30 minutes to compare different treatment conditions and different AGs below.

Mechanisms of AG delivery into lysosomes. A number of intracellular trafficking routes could deliver AGs to lysosomes. AGs could be captured from the extracellular space by endocytic carriers that fuse with lysosomes (18). Alternatively, AGs could be delivered from the cytosol to the lysosome by autophagic processes (59). To determine whether these general pathways are involved, we first asked whether endocytosis regulates AG lysosomal delivery. We monitored Neo-TR entry while inhibiting vesicle budding from the plasma membrane. The conserved GTPase dynamin forms a constrictive ring that releases vesicles from membranes, and is utilized by many endocytic processes $(60,61)$. Its activity can be 
A

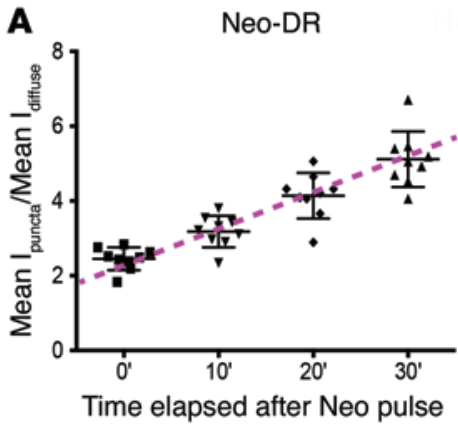

B

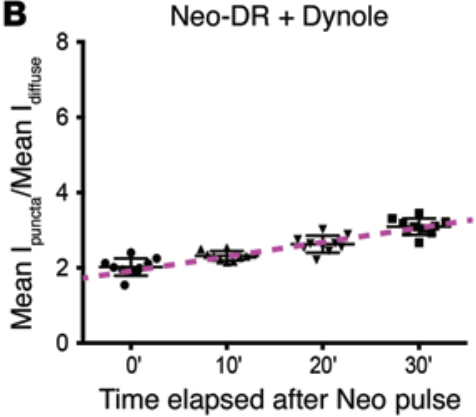

\section{C}
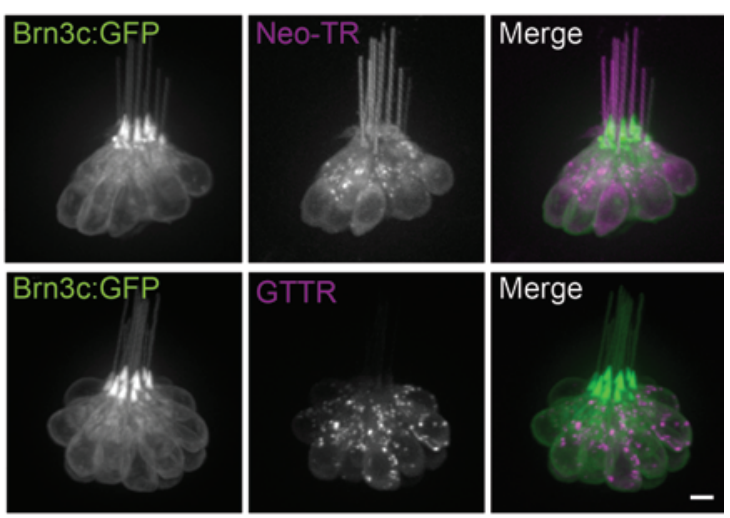
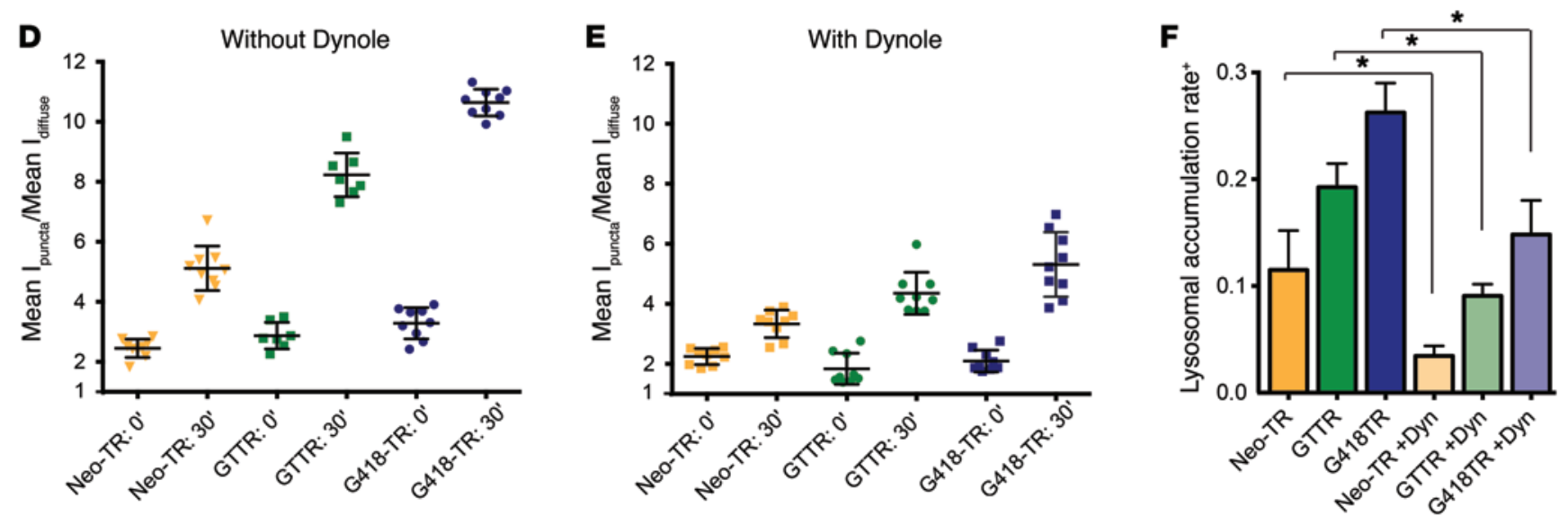

Figure 7. Endocytic inhibition delays lysosomal loading of 3 AGs. (A) Quantification of Neo-TR loading into lysosomes (method described in Figure 5C). Intensity within lysosomes is initially approximately 2.5 -fold higher in puncta compared with cytosol. This value increases to approximately 5 -fold after 30 minutes. Error bars: \pm 1 SD. Each graphed symbol in $\mathbf{A}$, B, D, and $\mathbf{E}$ represents 1 fish, 3 neuromasts per fish. (B) HCs treated with Dynole $34-2$ show reduced rates of Neo-TR delivery into lysosomes (compare with A). Error bars: \pm 1 SD. (C) Images of HCs exposed to a $50-\mu M$ pulse of either Neo-TR or Gent-TR (GTTR). Compared with Neo-TR, GTTR shows more signal in puncta, and less in the cytosol. Scale bar: $5 \mu \mathrm{m}$. (D) Quantification of delivery of labeled neomycin, gentamicin, and G418 to lysosomes immediately after pulse exposure and 30 minutes later. Error bars: \pm 1 SD. (E) HCs pretreated with Dynole $34-2$ show delayed delivery of neomycin, gentamicin, and G418 to lysosomes relative to HCs not treated with Dynole. Error bars: \pm 1 SD. (F) Summary plot of lysosomal loading rates for the 3 tested AGs, each with and without Dynole pretreatment. 'Graphed values indicate the change in relative amount of signal in lysosomes compared with the cytosol between 0 and 30 minutes (e.g., the slopes of purple dashed lines in $\mathbf{A}$ and $\mathbf{B}$ ). Error bars: \pm 1 SD for 3 replicates. ${ }^{*} P<0.05$ based on Mann-Whitney $U$ test.

blocked by Dynoles (Tocris Biosciences), a group of bisindolylmaleimides that specifically inhibit the GTPase activity of dynamin, and thereby its fission activity (62).

We pretreated zebrafish with $25 \mu \mathrm{M}$ Dynole $34-2$ for 5 minutes, and then exposed them to $50 \mu \mathrm{M}$ Neo-TR. In HCs imaged immediately after Neo-TR exposure, Neo-TR was present in the HCs, but puncta were almost completely absent (Figure 6A). The level of diffuse signal present in the cytosol was comparable to that seen in HCs not treated with Dynole. The specific loss of puncta but not cytosolic signal suggests that AGs entering HCs traverse at least 2 routes - a dynamin-sensitive and a dynamin-insensitive route. We further assessed the effect of Dynole 34-2 by evaluating loading of FM1-43, a readout for MET activity. As with NeoTR, Dynole 34-2 treatment blocks FM1-43 delivery to puncta, but not entry into the diffuse pool (Figure 6, B and C). The effect of Dynole treatment is strikingly different from the effect of the MET inhibitor amiloride. Amiloride treatment severely inhibits all FM1-43 loading. Furthermore, amiloride's effects are reversible, unlike those of Dynole. Dynole's inhibition of puncta does not appear to be due to effects via MET activity. This inhibition is dose dependent over a range from 6 to $25 \mu \mathrm{M}$ (Figure 6D). At $25 \mu \mathrm{M}$, almost no puncta can be seen, either when zebrafish are exposed to a pulse of Neo-TR immediately following the Dynole 34-2 treatment, or 15 minutes after the treatment (indicating that the effects of Dynole 34-2 are not readily reversible). We note that a different dynamin inhibitor - dynasore - does act like a MET inhibitor and blocks all FM1-43 and Neo-TR entry into HCs (see Figure 3B and Supplemental Figure 4).

Using Dynole 34-2, we evaluated how endocytosis contributes to the lysosomal delivery of AGs by comparing NeoTR delivery to lysosomes in untreated versus Dynole-treated HCs. Because the effects of Dynole are irreversible over the time scales needed for these experiments, we pretreated HCs with Dynole and then transferred fish to media with Neo-TR for pulse labeling. We then transferred pulse-labeled fish to fresh medium, and evaluated lysosome delivery rates. Neo-TR delivery into lysosomes is delayed when endocytosis is inhibited by Dynole 34-2 treatment (compare Figure 7A and Figure 


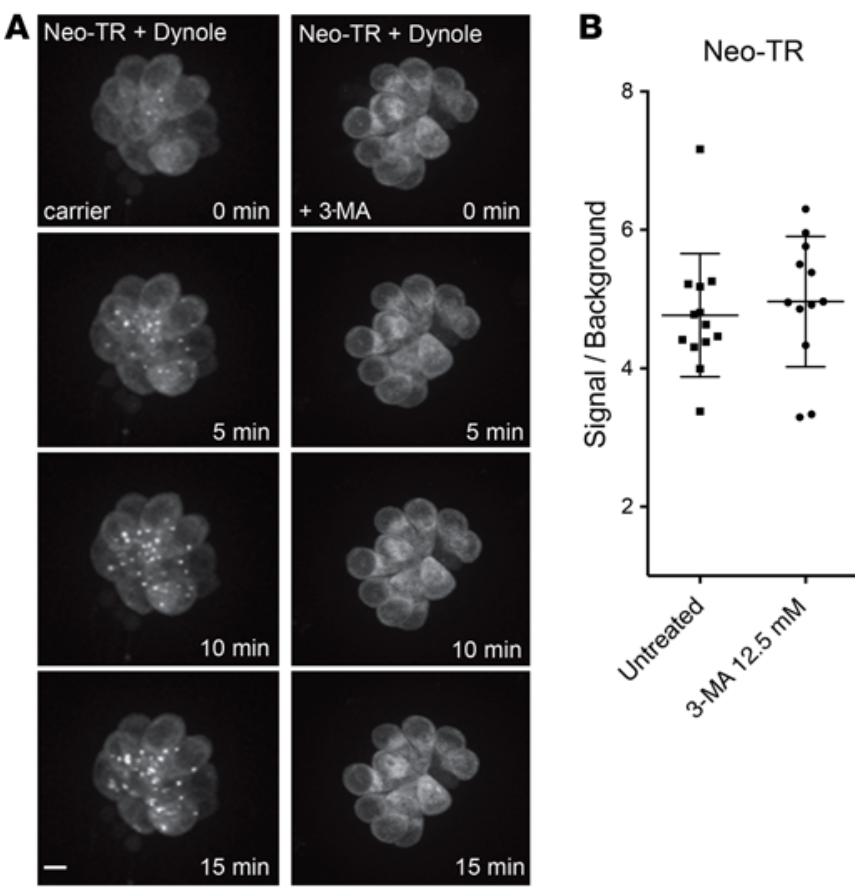

7B). These results demonstrate that AG entry into lysosomes is dependent on endocytosis, but over time AGs do accumulate in lysosomes in an endocytosis-independent manner.

We next asked whether the lysosomal delivery rates of structural variants of AGs are distinct. Pulse exposures of Neo-TR show a prominent diffuse signal in the cytoplasm (Figure 7C, top). Gent-TR, in contrast, shows a prominent signal in lysosomes and a less obvious diffuse pool (Figure 7C, bottom). To test whether this distribution reflects more efficient delivery to lysosomes, we quantified lysosomal delivery rates for both gentamicin and the structurally closely related AG G418, and compared these with the behavior of neomycin. Lysosomal delivery rates of both Gent-TR and G418-TR are significantly higher than rates for Neo-TR (Figure 7D). For all of these AGs, Dynole 34-2 treatment reduces the delivery rate to lysosomes over a 30-minute period (compare Figure 7D and Figure 7E; and see summary plot, Figure 7F). Different AG structures show different relative rates of accrual in diffuse and lysosomal compartments, and dependence on endocytic processes for delivery to the lysosome.

While Dynole 34-2 treatment abolishes puncta that appear rapidly after exposure to Neo-TR (within 5 minutes; Figure 6A), puncta do appear in the cytosol at later times. They are easily detected 20 minutes after the Neo-TR pulse in Dynole 34-2-treated HCs (Figure 8A, left panels). This is not due to reversibility of the Dynole 34-2 treatment. Exogenous Neo-TR added 15 minutes after Dynole 34-2 treatment and washout still does not rapidly appear in puncta in HCs imaged immediately after the pulse (Figure 6D). The later appearance of puncta indicates a lysosomal delivery route that is independent of dynamin-mediated endocytosis from the plasma membrane. This delivery also occurs in the absence of any extracellular Neo-TR because it occurs after washout of the Neo-TR pulse - likely indicating direct delivery from the cytosolic pool to lysosomes. We evaluated whether this delivery is via macroautophagy - the capture of components in
Figure 8. An autophagic pathway delivers Neo-TR to lysosomes and is independent of endocytosis. (A) HCs exposed to Dynole 34-2, washed out, and then exposed to Neo-TR in the absence or presence of the macroautophagy inhibitor 3-methyladenine (3-MA). In the absence of 3-MA, Neo-TR accumulates in lysosomes less than 5 minutes after the Neo-TR pulse (left panels). 3-MA abolishes the accumulation of Neo-TR in the lysosomes of HCs pretreated with Dynole 34-2 (right panels). Scale bar: $5 \mu \mathrm{m}$. (B) Treatment with 3-MA does not affect loading of Neo-TR. The loss of labeling in puncta is not due to an overall reduction in Neo-TR entry. Error bars: $\pm 1 \mathrm{SD}$. Each graphed symbol represents 1 fish, 5 neuromasts per fish.

the cytosol into autophagic vesicles that then fuse with lysosomes. 3-Methyladenine (3-MA), a PI3K inhibitor, is routinely used to inhibit macroautophagy (63) and has been used previously in live zebrafish (64). We found that 3-MA treatment dramatically blocks the slower delivery of AGs into lysosomes following a Neo-TR pulse exposure when dynamin activity is inhibited (Figure 8A, right panels). 3-MA treatment does not block rapid puncta formation if dynamin is not inhibited (not shown), nor does it affect the total amount of AGs loaded into HCs (Figure 8B). Based on these findings, it appears that autophagosomes capture AGs directly from the cytosol and fuse with lysosomes, and that this route is separate from the dynamin-dependent endocytic route.

Evaluating how lysosomal delivery of AGs affects HC toxicity. We next asked whether lysosomal delivery alters the toxicity of different AGs. Lysosomes can release pro-death signals $(65,66)$, or alternatively they can sequester molecules away from toxic targets. Previous work has shown that over short time frames (30-60 minutes), neomycin is more toxic to HCs than gentamicin (28). As shown above, Gent-TR is more efficiently delivered to lysosomes than Neo-TR (Figure 7F), suggesting that lysosomal capture is at least initially cytoprotective. To test this idea, we treated zebrafish with Dynole 34-2 to delay lysosomal delivery of neomycin or gentamicin, and evaluated how this affected the toxicity of these 2 AGs. Dose-response functions for zebrafish pretreated with Dynole revealed that delaying lysosomal delivery has little effect on the toxicity of neomycin (Figure 9A), but significantly increases the toxicity of gentamicin, most notably at higher concentrations (Figure 9B). We also compared the amount of labeled AG in the cytosol with and without Dynole 34-2 treatment. Dynole 34-2 treatment increases the amount of Gent-TR present in the cytosolic fraction 15 minutes after a pulse exposure by 16.1\% (Supplemental Figure 5). We do not detect an increase in the cytosolic fraction for Neo-TR, where the larger cytosolic pool may make the additional signal difficult to measure. These observations may explain why inhibiting endocytosis is more effective at altering the HC toxicity profile of gentamicin compared with neomycin.

Evaluating relationships between $M E T$ activity, HC loading rates, lysosomal delivery rates, and AG toxicity. Drugs that block FM1-43 loading, a proxy for MET activity, prevent entry of Neo-TR (Figure 3C). At lower concentrations, many of these drugs have intermediate effects. We next evaluated the effects of 1 drug - benzamil - a close structural relative of the epithelial sodium channel blocker amiloride that also inhibits MET $(67,68)$. Previous work shows that a $50-\mu \mathrm{M}$ pretreatment and coexposure to benzamil protects HCs from both neomycin and gentamicin (44). We evaluated NeoTR loading in HCs exposed to a range of benzamil concentrations that show partial to nearly complete block of Neo-TR loading -0 

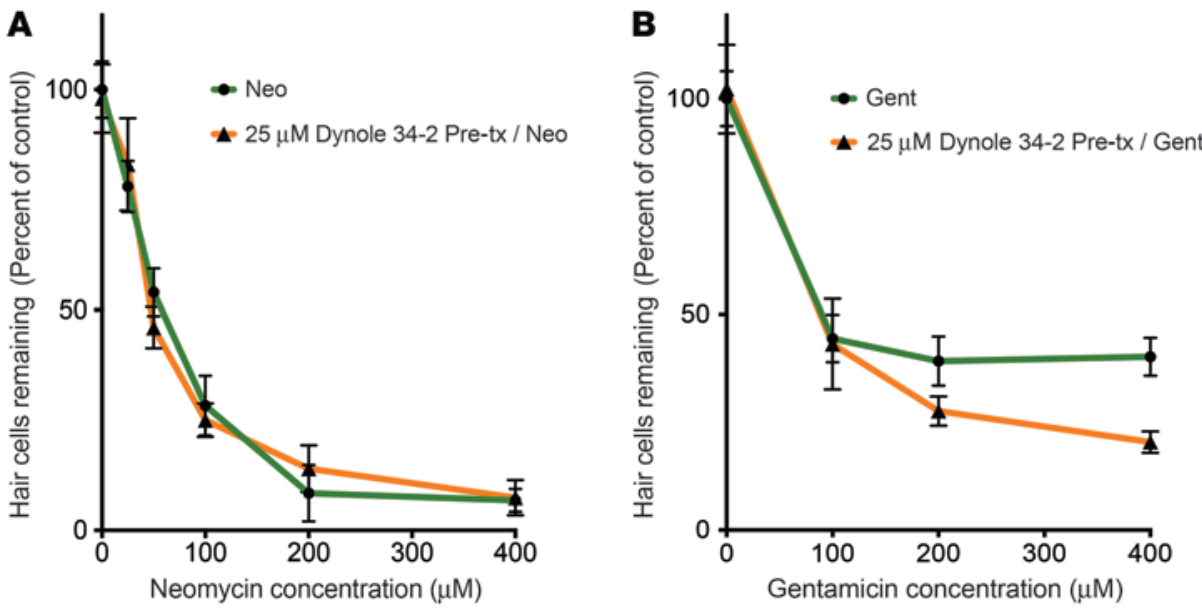

Figure 9. Inhibition of endocytosis differentially affects AG-induced HC death. (A) Dose-response functions for 1 hour neomycin exposure with and without Dynole 34-2 pretreatment. Inhibiting endocytosis before exposure to neomycin does not significantly affect $\mathrm{HC}$ survival in exposed zebrafish. (B) Dose-response functions for 1 hour gentamicin exposure with and without Dynole 34-2 pretreatment. In contrast to neomycin, gentamicin toxicity is affected. At higher concentrations of gentamicin, inhibiting endocytosis increases HC death. Error bars: \pm 1 SD. Each graphed symbol represent 5 fish, 5 neuromasts per fish. ANOVA $P<0.01$ for significance of Dynole treatment to protection from gentamicin. to $200 \mu \mathrm{M}$. In HCs pretreated for 5 minutes with $200 \mu \mathrm{M}$ benzamil and then cotreated with Neo-TR and benzamil, almost no Neo-TR is detectable (Figure 10A). At $25 \mu \mathrm{M}$ benzamil, the average total amount of loaded Neo-TR was reduced by roughly half in comparison with untreated HCs. To test how this range of benzamil concentrations also affects MET activity, we looked at this same dose-response function for FM1-43 uptake. Data for FM1-43 are more variable, but show a response similar to the Neo-TR uptake response (Figure 10B). We next looked at a narrower range of benzamil concentrations $(0-50 \mu \mathrm{M})$, and evaluated how incomplete reductions in Neo-TR loading affect the delivery of Neo-TR to lysosomes. Exposure to benzamil at concentrations that reduce but do not block either MET activity or Neo-TR loading appears to alter how Neo-TR transits different intracellular pathways. As the total amount of Neo-TR loading is reduced by benzamil, the relative amount of Neo-TR in lysosomes increases (Figure 10C). Fifty-micromolar benzamil treatment results in a roughly 2 -fold increase in the relative fraction in lysosomes compared with untreated cells. Lastly, we evaluated how this same concentration range of benzamil affects $\mathrm{HC}$ survival. There is a clear inverse relationship between the effects of benzamil on Neo-TR loading and on HC survival. These data suggest that reducing AG entry results in synergistic protective effects. Both overall amounts of AGs in HCs are lowered, and larger fractions of cytosolic AGs are sequestered in lysosomes. Both of these activities restrict AG access to cytotoxic targets.

\section{Discussion}

Understanding AG toxicity has proven challenging. AGs can interact with many cellular components (e.g., phospholipids in the plasma membrane, mitochondrial components, proteins in ER membranes, etc.) $(8,7,69)$. It is clear, however, that cellular entry is a key component of toxicity. AGs do not accumulate in most cell types, though inner ear HCs and kidney cells are exceptions $(30,70)$. Understanding AG entry and accumulation in HC compartments may therefore shed light on the cellular events underlying AG toxicity.

By imaging superficial HCs in living intact fish, we were able to monitor AG loading when the extracellular environment regulating ion flow was unperturbed, MET channels were functioning, and junctions with surrounding cells were intact. In these function- ing HCs, AGs preferentially enter mechanosensory hair cells of the lateral line (LL) system compared with other tissues. AGs enter apically through several processes that are all gated by MET. An endocytic route requires dynamin activity and rapidly delivers AGs to lysosomes. A route independent of dynamin-mediated endocytic events delivers AGs directly to the cytosol, presumably through the MET channel. A fraction of this cytosolic pool is also delivered to lysosomes through a PI3K-dependent, macroautophagic process. These data are summarized in the schematic in Figure 11.

Endocytosis and AG toxicity. In HCs, the role of endocytosis in AG entry is unclear. In the kidney (thought to lack the mechanosensitive channel present in HCs), the involvement of endocytic processes is well established. AGs in proximal tubule cells bind to megalin, a multiligand endocytic receptor (19). When exposed to gentamicin, the kidneys of megalin-deficient mice show severely reduced gentamicin levels. Megalin-deficient mice also exhibit hearing loss, but this has been attributed to effects on marginal cells of the stria vascularis; megalin expression is not seen in cochlear HCs (71). Previous studies have evaluated whether inhibiting endocytosis protects HCs, with the hypothesis that blocking this entry path will block overall AG entry and protect HCs (52). This prediction is consistent with work in the kidney, where AG endocytosis underlies AG toxicity (19). However, we show that in zebrafish LL HCs, endocytic processes rapidly sequester AGs into lysosomes. The fact that there is no change in overall loading when endocytosis is inhibited suggests that endocytic and nonendocytic routes compete. Consistent with this, the relative cytosolic fraction increases when HCs are exposed to higher AG concentrations. It appears therefore that the endocytic route can be overwhelmed. Our work suggests that endocytosis and lysosomal delivery sequester AGs away from cytotoxic targets. In this scenario, inhibiting endocytosis should increase AG toxicity, and we see this for gentamicin at short treatment times and high concentrations. Consistent with lysosomal delivery routes sequestering AGs from toxic targets, promoting lysosomal delivery through autophagy of AGs is cytoprotective in the kidney (72).

Role of MET in AG entry. A number of studies suggest that AG entry into inner ear hair cells occurs directly through the MET channel $(36,73)$. Delivery through the MET channel should not require endocytic machinery, and could account for the diffuse pool of cytosolic AGs we see in exposed HCs. Movement of AGs 


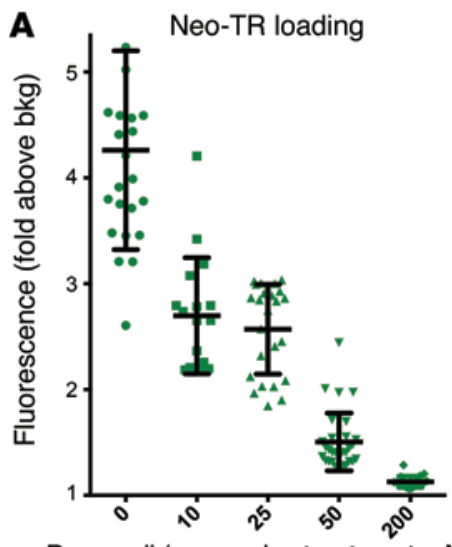

Benzamil (pre- and cotreatment; $\mu \mathrm{M}$ )

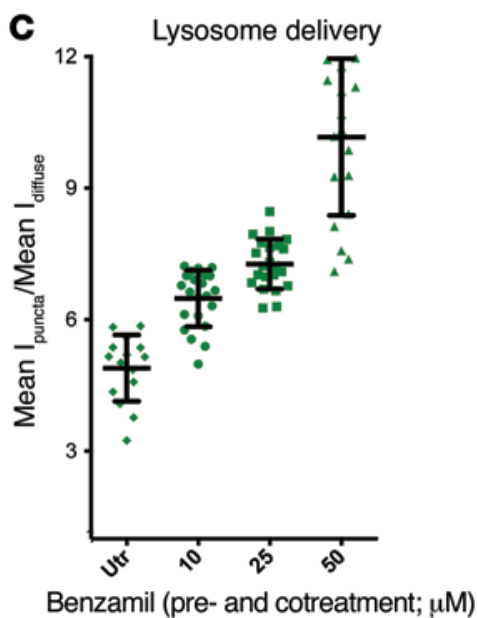

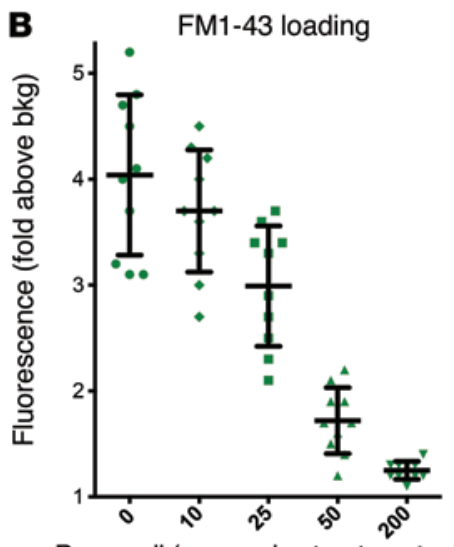

Benzamil (pre- and cotreatment; $\mu \mathrm{M}$ )

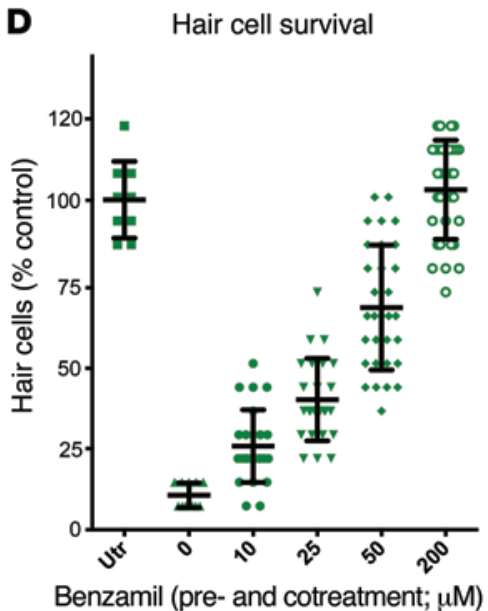

Figure 10. Inhibiting MET activity decreases the rate of AG loading into HCs, increases the relative amount of AGs delivered to lysosomes, and protects $\mathrm{HCs}$ from AG exposure. (A) Benzamil, an inhibitor of MET, impedes entry of Neo-TR into HCs in a dose-dependent manner. Zebrafish were pre-exposed for 5 minutes to indicated concentrations of benzamil, and then cotreated with $50 \mu \mathrm{M}$ Neo-TR for 5 minutes, washed, and imaged. Neo-TR signal was quantified as described in Figure 3A. (B) Treatment with benzamil also impedes FM1-43 entry into HCs in a dose-dependent manner, similarly to its analog amiloride, shown in Figure 3B, and consistent with its activity as a MET inhibitor. (C) The relative amount of Neo-TR loaded into lysosomes increases with increasing benzamil concentrations. Loading of Neo-TR into lysosomes was assayed as described in Figure 5C. (D) This same range of benzamil concentrations shows an increasing degree of $\mathrm{HC}$ protection from a 1-hour, 200- $\mu \mathrm{M}$ neomycin exposure. HC survival was assessed as described in Figure 1. Error bars: \pm 1 SD. Each graphed symbol represents 1 fish, 5 neuromasts per fish. directly through the MET channel readily explains why loss of functional MET activity protects HCs from AG exposure (20). However, it is not clear why loss of MET activity should prevent dynamin-dependent endocytic uptake of AGs, which we see as loss of punctate accumulations immediately after pulse exposure. Alterations in local calcium in the stereocilia could alter calcium-dependent endocytic processes. Alternatively there may be a direct movement of AGs from within the channel into membranes that are then endocytosed. Notably, though, AGs are present in the lumen of lysosomes and do not appear to be enriched on lysosomal membranes (where they would be predicted to arrive if they moved to the lysosome through membrane fusion events), and the charge of AGs does not favor this scenario. It is also possible that there are a class of apical endosomes that rapidly acquire AGs after passage through the channel that could be sensitive to Dynole 34-2 treatment. However, the effect of Dynole 34-2 treatment is nearly instantaneous, so presumably these endosomes would have to be very dynamic to be sensitive at that time scale.

Many studies report that AGs themselves physically block the channel and impede MET activity - inhibiting electrophysiological responses of stimulated HCs $(36,73,74)$. When we expose LL HCs to AG concentrations that do not cause obvious HC damage, we do not observe inhibition of further AG loading. In fact, at noncytotoxic concentrations, AGs in LL HCs appear to promote their own entry. Exposed HCs show increased loading rates both as exposure time increases, and as concentration increases; that is, first derivatives of loading curves have positive slopes.

A number of alternative entry mechanisms have been proposed in addition to the MET channel or through endocytosis. It has been suggested that AGs enter through other channels including transient receptor potential channels (75-77). The sodium glucose transporter SLC5A2 may also play a role in AG transport in the kidney (78). Whatever additional mechanisms of uptake are active, our data suggest that these mechanisms are universally under control of MET activity in hair cells.

Mechanism of protection of small-molecule protectants. Having characterized the routes of AG entry, we asked how protective molecules alter these processes. A suite of unrelated compounds block both MET activity and AG entry. These molecules span structures that do not share known targets or obvious chemical characteristics. Channels passing AGs are broadly permissive to disparate kinds of molecules that impede normal flux (79); these molecules may compete with AGs for channel access. Another possibility is that the molecules perturb lipid tension in the apical region. The architecture of stereocilia may maximize conversion of mechanical movement into membrane deformation. Altering lipid tension where channels reside could regulate their activity, as has been reported for other channel proteins (80). AG entry could then be especially sensitive to molecules that interact with the apical lipid bilayer, not necessarily just the channel itself (81). At this 


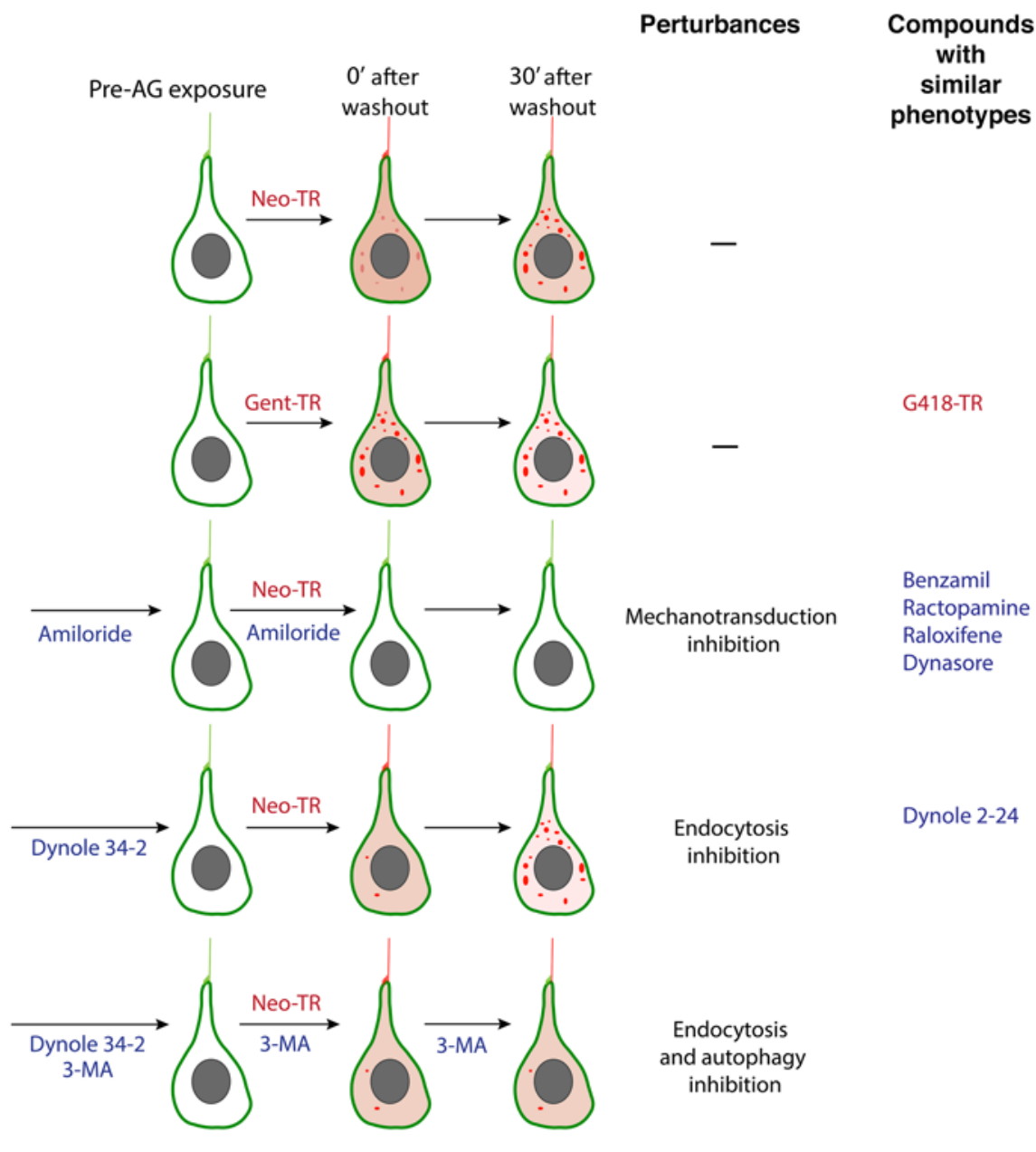

Figure 11. Schematic summarizing entry and distribution of AGs in live LL HCs, and the effects of compounds that alter these processes. AGs initially appear in the apical region, first labeling stereocilia, and kinocilia. They next appear in both diffuse and punctate intracellular pools. Different AGs exhibit different delivery rates to these different pools. When MET activity is blocked (e.g., by pretreatment and cotreatment with amiloride), all AGs fail to enter HCs. When dynamin-dependent endocytosis is inhibited (e.g., by Dynole 34-2 pretreatment), rapid delivery of AGs to the cytosol still occurs, but AG-labeled puncta are initially absent. Puncta do appear later. These late-forming puncta do not appear when both dynamin-dependent endocytosis and macroautophagy are inhibited (by pretreatment with Dynole 34-2 and pre- and cotreatment with the macroautophagy inhibitor 3-MA).

time, the mechanism is unclear, but MET activity is certainly perturbed by many compounds, and when it is blocked, LL HCs are protected from AG exposure. AGs then do not enter HCs, through either endocytic or nonendocytic routes.

When AG loading is reduced but not blocked, how protection occurs is more complicated. We previously identified both drug treatments and genetic mutations that impede AG entry $(42,82$, 83). Here we show that reducing the total amount of AGs entering the cell also results in a larger relative fraction of AGs being delivered to the lysosomal compartment. Furthermore, this lysosomal delivery appears to be cytoprotective, at least in short-term experiments. Using approaches described here, we could potentially quantify lysosomal delivery rates for many different AGs. These assays may help classify which AGs are less damaging to HCs.

In further studies, it would be interesting to assess the long-term consequences of AG sequestration in lysosomes. LL HCs that are exposed to a nontoxic pulse of Neo-TR still have signal in lysosomes 7 days after washout (Supplemental Figure 6). This is consistent with our quantitative studies and others showing that labeled AGs are not readily exported from HCs (84). HCs exposed continuously for 7 days to nontoxic neomycin levels $(20 \mu \mathrm{M})$ look morphologically normal (Supplemental Figure 6) and have active MET based on their uptake of FM1-43. However, effects of chronic lysosome accumulation will need to be further evaluated over longer time courses in systems that do not replace HCs.
Implications for other studies. In the mammalian inner ear, punctate accumulations of AGs have been observed in many studies, although they have not been extensively characterized. In mice exposed to systemic Gent-TR, fluorescent AGs appear to transit vestibular vasculature to first reach dark and transitional cells, where they appear as very bright puncta (defined in this study as $6^{+}$-pixel structures that exceed the $99 \%$ quantile of fluorescence intensity). Puncta are evident within 30 minutes of systemic exposure (85). In sensory hair cells, fluorescent signal is predominantly diffuse, but localized accumulations are present. Notably, after exposure, fluorescent Gent-TR can persist in transitional cells for more than 28 days - likely trapped in lysosomes (86). The details of how AGs reach sensory cells and the kinetics of their entry into those cells are complicated in the mammalian ear. Here we show that zebrafish studies can identify likely intracellular trafficking routes transited in sensory hair cells.

This work also demonstrates more broadly how labeling a compound of interest and following its behavior at high resolution in live differentiated tissues can potentially inform us about how the compound behaves in a specific physiological context. Such studies can identify cellular processes in specific cell types that may provide tractable targets for combinatorial drug therapies. High-resolution studies can reveal processes that can be targeted in order to either enhance or restrict cell entry and metabolic processing (here, the separable dynamin-depen- 
dent endocytic, nonendocytic, and autophagic routes). The activity of labeled compounds needs to be validated if they are being used as proxies for unlabeled compounds. However, labeling frequently does not disrupt activity of the target molecule, and many compounds of interest are fluorescent themselves. Advances in both labeling technologies and live-imaging modalities will continue to improve our ability to evaluate how cells respond to bioactive compounds.

\section{Methods}

Fish. Adult Danio rerio (zebrafish) were maintained in the University of Washington zebrafish facility. Experiments were carried out on zebrafish 5 days post fertilization (dpf). Zebrafish were maintained in E3 embryo media $\left(14.97 \mathrm{mM} \mathrm{NaCl}, 500 \mu \mathrm{M} \mathrm{KCl}, 42 \mu \mathrm{M} \mathrm{Na}_{2} \mathrm{HPO}_{4}, 150\right.$ $\mu \mathrm{M} \mathrm{KH}_{2} \mathrm{PO}_{4}, 1 \mathrm{mM} \mathrm{CaCl}_{2}$ dihydrate, $1 \mathrm{mM} \mathrm{MgSO}_{4}, 0.714 \mathrm{mM} \mathrm{NaHCO}_{3}$, $\mathrm{pH}$ 7.2) unless otherwise noted. The following genetic lines were used:

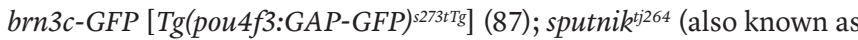
$\left.c d h 23^{\text {t264 }}\right)(35)$; and myo6-GFP (myo6b:eGFP'186). GFP-Rab7 fish were provided by the L. Ramakrishnan laboratory (Cambridge University, Cambridge, United Kingdom). They express the EGFP-Rab7 ORF from the Brian Link laboratory (Medical College of Wisconsin, Milwaukee, Wisconsin, USA). sputnik ${ }^{\text {t264 }}$ fish were provided by T. Nicolson (Oregon Health and Science University, Portland, Oregon, USA).

Transgenesis constructs. Standard Gateway (Invitrogen) cloning techniques were used to generate transgenesis constructs under the control of either the $\mathrm{HC}$-specific myosin $6 \mathrm{~b}$ promoter (88) or the $\beta$-actin (actb2) promoter (38).

Vital dye staining. Live swimming zebrafish were incubated in the indicated vital dyes, washed 3 times in embryo media, and immobilized for imaging as described below. MitoTracker Green FM (Life Technologies) was used at $100 \mathrm{nM}$ for 3 minutes. LysoTracker Green DND-26 (Life Technologies) was used at $50 \mathrm{nM}$ for 3 minutes. FM1-43 was used at $500 \mathrm{nM}$ for 5 minutes.

Imaging. $5 \mathrm{dpf}$ zebrafish were placed on their side in chambered coverglass wells containing E3 media with $0.2 \%$ MESAB (tricaine; MS-222; ethyl-m-aminobenzoate methanesulfonate). Fine nylon mesh and a brain slice anchor harp (Harvard Instruments) were placed over sedated zebrafish for stability during imaging. Imaged neuromasts on immobilized zebrafish had free access to surrounding media. Image stacks were captured at 16-bit depth with a Marianas spinning disk system (Intelligent Imaging Innovations) equipped with an Evolve 10-MHz EMCCD camera (Photometrics), Zeiss C-Apochromat $\times 63 / 1.2 \mathrm{NA}$ Water Objective, and a piezoelectric motorized stage (ASI). Camera intensifications were set to achieve exposure times less than $50 \mathrm{~ms}$ and maximum pixel intensities less than $75 \%$ of saturation, and were held constant across any experiments that were directly compared. Tests were run to confirm linear sensitivity of the camera over detected intensity ranges. Image volumes were acquired at $0.8-\mu \mathrm{m}$ intervals over $25 \mu \mathrm{m}$ along the $z$ axis (greater than the depth of the neuromast). GFP, green dyes, and FM1-43 fluorescence were acquired using $490 \mathrm{~nm}$ excitation and $535 / 30$ emission. Texas red fluorescence was acquired using $561 \mathrm{~nm}$ excitation and 617/73 emission.

$A G$ pulse exposure. Live swimming zebrafish were transferred to wells containing indicated concentrations of labeled AGs. After the incubation period, zebrafish were pipette-transferred to 3 sequential wells containing $\mathrm{E} 3$ media for 30 seconds in each, and finally transferred to an imaging chamber containing E3/0.2\% MESAB. Neuromasts were immediately located by eye with transmitted differential interference contrast (DIC), and fluorescent images were then captured.

Quantification of AG uptake. To quantify total AG loading into HCs, we wrote a MATLAB script that opens 3D 2-channel 16-bit image stacks, identifies the HC volume (by identifying green signal in myo6GFP-expressing fish), extracts the red (AG-Texas red) signal in this $\mathrm{HC}$ volume, and finally compares the mean AG-Texas red signal with the mean background signal (extracted from the region without myo6GFP signal). The script uses Otsu analysis to automatically determine a thresholding value that identifies HCs, and then uses this to generate a binary mask. Analyzed image stacks each contain 1 neuromast. At least 5 neuromasts were analyzed from at least 5 fish. Data are shown as average signal/background with standard deviations. Analyses were run in MATLAB version R2014b.

Quantification of lysosomal delivery. To quantify the rate of AG delivery to lysosomes, 5-day-old zebrafish were exposed to a pulse of $20 \mu \mathrm{M}$ Neo-BODIPY for 3 minutes and washed 3 times in E3 media. Zebrafish were maintained in E3 for 30 minutes, exposed to $25 \mu \mathrm{M}$ Neo-TR for 5 minutes, and then washed 3 times in E3. Image stacks were taken (as described for AG loading) at the indicated times following the Neo-TR pulse exposure. Neo-BODIPY-labeled lysosomes were identified by Otsu thresholding of the green channel; this signal was used to define an image mask. Red signal from Neo-TR was then quantified in this region, as well as in the image outside of this region but diffusely in the HCs.

Figure preparation. Figures were prepared using Adobe Photoshop, Illustrator CS6, and GraphPad Prism 7.

Statistics. Statistical analyses were done using GraphPad Prism 7.0. Specific analyses are indicated in the figure legends. Kruskal-Wallis analysis followed by Holm-Šidák multiple comparison tests was used to compare indicated sets. ANOVA was used to evaluate dose responses. $P$ values less than 0.05 were considered significant.

Study approval. Experiments were approved by the University of Washington Institution Animal Care and Use Committee.

\section{Author contributions}

DWH, RE, THL, EWR, and DWR designed the studies. DWH and THL conducted experiments. DWH, RE, and DWR analyzed data. DWH, EWR, and DWR wrote the manuscript.

\section{Acknowledgments}

The authors thank David White for fish care and Lalita Ramakrishnan, Brian Link, and Teresa Nicolson for the zebrafish lines (described in Methods). The Open Microscopy Environment (OME) provides downloadable MATLAB scripts to import 4D 16-bit OME formatted files into the MATLAB environment. We called these subroutines in our script to analyze fluorescent AG loading into HCs. MATLAB "bioformats" scripts are available at https://www.openmicroscopy.org/site/products/bio-formats. Work was supported by National Institute on Deafness and Other Communication Disorders grants DC012881 and DC05987.

Address correspondence to: David W. Raible, University of Washington, Department of Biological Structure, Health Sciences Building H-501, 1959 NE Pacific Street, Seattle, Washington 981957420, USA. Phone: 206.616.1048; E-mail: draible@uw.edu. 
1. Vakulenko SB, Mobashery S. Versatility of aminoglycosides and prospects for their future. Clin Microbiol Rev. 2003;16(3):430-450.

2. Polin RA, Committee on Fetus Newborn. Management of neonates with suspected or proven early-onset bacterial sepsis. Pediatrics. 2012;129(5):1006-1015

3. Falagas ME, Matthaiou DK, Bliziotis IA. The role of aminoglycosides in combination with a $\beta$-lactam for the treatment of bacterial endocarditis: a meta-analysis of comparative trials.

JAntimicrob Chemother. 2006;57(4):639-647.

4. Davies J, Davis BD. Misreading of ribonucleic acid code words induced by aminoglycoside antibiotics. The effect of drug concentration. J Biol Chem. 1968;243(12):3312-3316.

5. Cabañas MJ, Vázquez D, Modolell J. Inhibition of ribosomal translocation by aminoglycoside antibiotics. Biochem Biophys Res Commun. 1978;83(3):991-997.

6. Misumi M, Nishimura T, Komai T, Tanaka N. Interaction of kanamycin and related antibiotics with the large subunit of ribosomes and the inhibition of translocation. Biochem Biophys Res Commun. 1978;84(2):358-365.

7. Qian Y, Guan MX. Interaction of aminoglycosides with human mitochondrial 12S rRNA carrying the deafness-associated mutation. Antimicrob Agents Chemother. 2009;53(11):4612-4618.

8. Brasseur R, Laurent G, Ruysschaert JM, Tulkens P. Interactions of aminoglycoside antibiotics with negatively charged lipid layers. Biochemical and conformational studies. Biochem Pharmacol. 1984;33(4):629-637.

9. Marche P, Koutouzov S, Girard A. Impairment of membrane phosphoinositide metabolism by aminoglycoside antibiotics: streptomycin, amikacin, kanamycin, dibekacin, gentamicin and neomycin. JPharmacol Exp Ther. 1983;227(2):415-420.

10. Pirvola U, et al. Rescue of hearing, auditory hair cells, and neurons by CEP-1347/KT7515, an inhibitor of c-Jun $\mathrm{N}$-terminal kinase activation. JNeurosci. 2000;20(1):43-50.

11. Coffin AB, Williamson KL, Mamiya A, Raible DW, Rubel EW. Profiling drug-induced cell death pathways in the zebrafish lateral line. Apoptosis. 2013;18(4):393-408.

12. Cheng AG, Cunningham LL, Rubel EW. Hair cell death in the avian basilar papilla: characterization of the in vitro model and caspase activation. JAssoc Res Otolaryngol. 2003;4(1):91-105.

13. Cunningham LL, Cheng AG, Rubel EW. Caspase activation in hair cells of the mouse utricle exposed to neomycin. J Neurosci. 2002;22(19):8532-8540.

14. Jiang H, Sha SH, Forge A, Schacht J. Caspaseindependent pathways of hair cell death induced by kanamycin in vivo. Cell Death Differ. 2006;13(1):20-30.

15. Esterberg R, Hailey DW, Rubel EW, Raible DW. ER-mitochondrial calcium flow underlies vulnerability of mechanosensory hair cells to damage. J Neurosci. 2014;34(29):9703-9719.

16. Esterberg R, et al. Mitochondrial calcium uptake underlies ROS generation during aminoglycoside-induced hair cell death. J Clin Invest. 2016;126(9):3556-3566.
17. Huth ME, Ricci AJ, Cheng AG. Mechanisms of aminoglycoside ototoxicity and targets of hair cell protection. Int J Otolaryngol. 2011;2011:937861.

18. Hashino E, Shero M. Endocytosis of aminoglycoside antibiotics in sensory hair cells. Brain Res. 1995;704(1):135-140.

19. Nagai J, Takano M. Entry of aminoglycosides into renal tubular epithelial cells via endocytosisdependent and endocytosis-independent pathways. Biochem Pharmacol. 2014;90(4):331-337.

20. Alharazneh A, et al. Functional hair cell mechanotransducer channels are required for aminoglycoside ototoxicity. PLoS One. 2011;6(7):e22347.

21. Stawicki TM, Esterberg R, Hailey DW, Raible DW, Rubel EW. Using the zebrafish lateral line to uncover novel mechanisms of action and prevention in drug-induced hair cell death. Front Cell Neurosci. 2015;9:46.

22. Raible DW, Kruse GJ. Organization of the lateral line system in embryonic zebrafish. JComp Neurol. 2000;421(2):189-198.

23. Thomas ED, Cruz IA, Hailey DW, Raible DW. There and back again: development and regeneration of the zebrafish lateral line system. Wiley Interdiscip Rev Dev Biol. 2015;4(1):1-16.

24. Esterberg R, Coffin AB, Ou H, Simon JA, Raible DW, Rubel EW. Fish in a dish: drug discovery for hearing habilitation. Drug Discov Today Dis Models. 2013;10(1):10.1016/j.ddmod.2012.02.001.

25. Hernández PP, Moreno V, Olivari FA, Allende ML. Sub-lethal concentrations of waterborne copper are toxic to lateral line neuromasts in zebrafish (Danio rerio). Hear Res. 2006;213(1-2):1-10.

26. Linbo TL, Stehr CM, Incardona JP, Scholz NL. Dissolved copper triggers cell death in the peripheral mechanosensory system of larval fish. Environ Toxicol Chem. 2006;25(2):597-603.

27. Ou HC, Raible DW, Rubel EW. Cisplatin-induced hair cell loss in zebrafish (Danio rerio) lateral line. Hear Res. 2007;233(1-2):46-53.

28. Owens KN, Coffin AB, Hong LS, Bennett KO, Rubel EW, Raible DW. Response of mechanosensory hair cells of the zebrafish lateral line to aminoglycosides reveals distinct cell death pathways. Hear Res. 2009;253(1-2):32-41.

29. Huth ME, et al. Designer aminoglycosides prevent cochlear hair cell loss and hearing loss. J Clin Invest. 2015;125(2):583-592.

30. Wang Q, Steyger PS. Trafficking of systemic fluorescent gentamicin into the cochlea and hair cells. JAssoc Res Otolaryngol. 2009;10(2):205-219.

31. Dai CF, Steyger PS. A systemic gentamicin pathway across the stria vascularis. Hear Res. 2008;235(1-2):114-124.

32. Harris JA, Cheng AG, Cunningham LL, MacDonald G, Raible DW, Rubel EW. Neomycin-induced hair cell death and rapid regeneration in the lateral line of zebrafish (Danio rerio). J Assoc Res Otolaryngol. 2003;4(2):219-234.

33. Owens KN, Coffin AB, Hong LS, Bennett KO, Rubel EW, Raible DW. Response of mechanosensory hair cells of the zebrafish lateral line to aminoglycosides reveals distinct cell death pathways. Hear Res. 2009;253(1-2):32-41.

34. Seiler C, Nicolson T. Defective calmodulin-dependent rapid apical endocytosis in zebrafish sensory hair cell mutants. J Neurobiol. 1999;41(3):424-434.

35. Söllner C, et al. Mutations in cadherin 23 affect tip links in zebrafish sensory hair cells. Nature. 2004;428(6986):955-959.

36. Marcotti W, van Netten SM, Kros CJ. The aminoglycoside antibiotic dihydrostreptomycin rapidly enters mouse outer hair cells through the mechano-electrical transducer channels.

JPhysiol (Lond). 2005;567(Pt 2):505-521.

37. Stawicki TM, et al. Cilia-associated genes play differing roles in aminoglycoside-induced hair cell death in zebrafish. G3 (Bethesda). 2016;6(7):2225-2235

38. Kwan KM, et al. The Tol2kit: a multisite gateway-based construction kit for Tol2 transposon transgenesis constructs. Dev Dyn. 2007;236(11):3088-3099.

39. Otsu N. A threshold selection method method from gray-level histograms. IEEE Trans Syst Man Cybern. 1979;9:62-66.

40. Ernest S, Rauch GJ, Haffter P, Geisler R, Petit C, Nicolson T. Mariner is defective in myosin VIIA: a zebrafish model for human hereditary deafness. Hum Mol Genet. 2000;9(14):2189-2196.

41. Rüsch A, Kros CJ, Richardson GP. Block by amiloride and its derivatives of mechano-electrical transduction in outer hair cells of mouse cochlear cultures. J Physiol (Lond). 1994;474(1):75-86.

42. Owens KN, et al. Identification of genetic and chemical modulators of zebrafish mechanosensory hair cell death. PLoS Genet. 2008;4(2):e1000020.

43. Ou HC, et al. Identification of FDA-approved drugs and bioactives that protect hair cells in the zebrafish (Danio rerio) lateral line and mouse (Mus musculus) utricle. J Assoc Res Otolaryngol. 2009;10(2):191-203.

44. Vlasits AL, Simon JA, Raible DW, Rubel EW, Owens KN. Screen of FDA-approved drug library reveals compounds that protect hair cells from aminoglycosides and cisplatin. Hear Res. 2012;294(1-2):153-165.

45. Gale JE, Marcotti W, Kennedy HJ, Kros CJ, Richardson GP. FM1-43 dye behaves as a permeant blocker of the hair-cell mechanotransducer channel. J Neurosci. 2001;21(18):7013-7025.

46. Sandoval R, Leiser J, Molitoris BA. Aminoglycoside antibiotics traffic to the Golgi complex in LLC-PK1 cells. JAm Soc Nephrol. 1998;9(2):167-174.

47. Sandoval RM, Molitoris BA. Gentamicin traffics retrograde through the secretory pathway and is released in the cytosol via the endoplasmic reticulum. Am J Physiol Renal Physiol. 2004;286(4):F617-F624.

48. Lippincott-Schwartz J, Yuan LC, Bonifacino JS, Klausner RD. Rapid redistribution of Golgi proteins into the ER in cells treated with brefeldin A: evidence for membrane cycling from Golgi to ER. Cell. 1989;56(5):801-813.

49. Doherty GJ, McMahon HT. Mechanisms of endocytosis. Annu Rev Biochem. 2009;78:857-902.

50. Mayor S, Pagano RE. Pathways of clathrinindependent endocytosis. Nat Rev Mol Cell Biol. 2007;8(8):603-612.

51. Toshima JY, et al. Bifurcation of the endocytic pathway into Rab5-dependent and -independent transport to the vacuole. Nat Commun. 
2014;5:3498.

52. Hashino E, Shero M, Salvi RJ. Lysosomal targeting and accumulation of aminoglycoside antibiotics in sensory hair cells. Brain Res. 1997;777(1-2):75-85.

53. Hirose K, Westrum LE, Stone JS, Zirpel L, Rubel EW. Dynamic studies of ototoxicity in mature avian auditory epithelium. Ann N Y Acad Sci. 1999;884:389-409.

54. Hirose K, Westrum LE, Cunningham DE, Rubel EW. Electron microscopy of degenerative changes in the chick basilar papilla after gentamicin exposure. JComp Neurol. 2004;470(2):164-180.

55. Abreu BJ, et al. Protein kinase $\mathrm{C}$ modulates synaptic vesicle acidification in a ribbon type nerve terminal in the retina. Neurochem Int. 2008;53(5):155-164.

56. Clark BS, Winter M, Cohen AR, Link BA. Generation of Rab-based transgenic lines for in vivo studies of endosome biology in zebrafish. Dev Dyn. 2011;240(11):2452-2465.

57. Humphries WH, Szymanski CJ, Payne CK. Endo-lysosomal vesicles positive for Rab7 and LAMP1 are terminal vesicles for the transport of dextran. PLoS One. 2011;6(10):e26626.

58. Tulkens P, Trouet A. The uptake and intracellular accumulation of aminoglycoside antibiotics in lysosomes of cultured rat fibroblasts. Biochem Pharmacol. 1978;27(4):415-424.

59. Klionsky DJ, Emr SD. Autophagy as a regulated pathway of cellular degradation. Science. 2000;290(5497):1717-1721.

60. McNiven MA. Dynamin: a molecular motor with pinchase action. Cell.1998;94(2):151-154.

61. Ferguson SM, De Camilli P. Dynamin, a membrane-remodelling GTPase. Nat Rev Mol Cell Biol. 2012;13(2):75-88.

62. Hill TA, et al. Inhibition of dynamin mediated endocytosis by the dynoles - synthesis and functional activity of a family of indoles. JMed Chem. 2009;52(12):3762-3773.

63. Seglen PO, Gordon PB. 3-Methyladenine: specific inhibitor of autophagic/lysosomal protein degradation in isolated rat hepatocytes. Proc Natl Acad Sci US A. 1982;79(6):1889-1892.

64. He C, Bartholomew CR, Zhou W, Klionsky DJ. Assaying autophagic activity in transgenic GFP-
Lc3 and GFP-Gabarap zebrafish embryos. Autophagy. 2009;5(4):520-526.

65. Mrschtik M, Ryan KM. Lysosomal proteins in cell death and autophagy. FEBS J. 2015;282(10):1858-1870.

66. Aits S, Jäättelä M. Lysosomal cell death at a glance. J Cell Sci. 2013;126(Pt 9):1905-1912.

67. Ricci A. Differences in mechano-transducer channel kinetics underlie tonotopic distribution of fast adaptation in auditory hair cells. J Neurophysiol. 2002;87(4):1738-1748.

68. Hirsh AJ, et al. Evaluation of second generation amiloride analogs as therapy for cystic fibrosis lung disease. J Pharmacol Exp Ther. 2004;311(3):929-938.

69. Karasawa T, Wang Q, David LL, Steyger PS. CLIMP-63 is a gentamicin-binding protein that is involved in drug-induced cytotoxicity. Cell Death Dis. 2010;1:e102.

70. Nilsson Tammela M, Tjälve H. Whole-body autoradiography of $[3 \mathrm{H}]$ dihydrostreptomycin in guinea pigs and rats: the labelling of the inner ear in relation to other tissues. Acta Otolaryngol. 1986;101(3-4):247-256.

71. König O, et al. Estrogen and the inner ear: megalin knockout mice suffer progressive hearing loss. FASEB J. 2008;22(2):410-417.

72. Cui J, et al. Rapamycin protects against gentamicin-induced acute kidney injury via autophagy in mini-pig models. Sci Rep. 2015;5:11256.

73. Kroese AB, Das A, Hudspeth AJ. Blockage of the transduction channels of hair cells in the bullfrog's sacculus by aminoglycoside antibiotics. Hear Res. 1989;37(3):203-217.

74. Kimitsuki T, Ohmori H. Dihydrostreptomycin modifies adaptation and blocks the mechanoelectric transducer in chick cochlear hair cells. Brain Res. 1993;624(1-2):143-150.

75. Myrdal SE, Steyger PS. TRPV1 regulators mediate gentamicin penetration of cultured kidney cells. Hear Res. 2005;204(1-2):170-182.

76. Karasawa T, Wang Q, Fu Y, Cohen DM, Steyger PS. TRPV4 enhances the cellular uptake of aminoglycoside antibiotics. J Cell Sci. 2008;121(pt 17):2871-2879.

77. Stepanyan RS, et al. TRPA1-mediated accumulation of aminoglycosides in mouse cochlear outer hair cells. JAssoc Res Otolaryngol. 2011;12(6):729-740.

78. Jiang M, Wang Q, Karasawa T, Koo JW, Li H, Steyger PS. Sodium-glucose transporter-2 (SGLT2; SLC5A2) enhances cellular uptake of aminoglycosides. PLoS One. 2014;9(9):e108941.

79. Ohmori H. Ion channels for the mechano-electrical transduction and efferent synapse of the hair cell. Adv Biophys. 1992;28:1-30.

80. Hamill OP, Martinac B. Molecular basis of mechanotransduction in living cells. Physiol Rev. 2001;81(2):685-740.

81. Kim J. Unconventional mechanics of lipid membranes: a potential role for mechanotransduction of hair cell stereocilia. Biophys J. 2015;108(3):610-621

82. Hailey DW, et al. Loss of Slc4a1b chloride/bicarbonate exchanger function protects mechanosensory hair cells from aminoglycoside damage in the zebrafish mutant persephone. PLoS Genet. 2012;8(10):e1002971.

83. Stawicki TM, Owens KN, Linbo T, Reinhart KE, Rubel EW, Raible DW. The zebrafish merovingian mutant reveals a role for $\mathrm{pH}$ regulation in hair cell toxicity and function. Dis Model Mech. 2014;7(7):847-856.

84. Hiel H, Bennani H, Erre JP, Aurousseau C, Aran JM. Kinetics of gentamicin in cochlear hair cells after chronic treatment. Acta Otolaryngol. 1992;112(2):272-277.

85. Liu J, Kachelmeier A, Dai C, Li H, Steyger PS. Uptake of fluorescent gentamicin by peripheral vestibular cells after systemic administration. PLoS One. 2015;10(3):e0120612.

86. Zhang R, Zhang YB, Dai CF, Steyger PS. Temporal and spatial distribution of gentamicin in the peripheral vestibular system after transtympanic administration in guinea pigs. Hear Res. 2013;298:49-59.

87. Xiao T, Roeser T, Staub W, Baier H. A GFP-based genetic screen reveals mutations that disrupt the architecture of the zebrafish retinotectal projection. Development. 2005;132(13):2955-2967.

88. Obholzer N, et al. Vesicular glutamate transporter 3 is required for synaptic transmission in zebrafish hair cells. J Neurosci. 2008;28(9):2110-2118. 